\title{
LWIR Snapshot Imaging Polarimeter
}

\author{
Dr. Robert E Sampson
}

I Technology Applications

2663 Wayside Dr.

Ann Arbor, MI 48103

Subcontractor: Optical Sciences Center

University of Arizona

April, 2009

Department of Energy

Technical Topic: 44 Remote Sensing

Subtopic: a. Polarimetry in Remote Sensing Applications

Phase 1 Grant Award Number: DE-FG02-08ER86346 


\section{Executive Summary}

Polarization images can provide a higher degree of contrast than an intensity image, providing the opportunity for dramatically improved object identification ${ }^{1}$. Furthermore, incorporation of a polarimeter allows for the potential to ascertain the Stokes vector elements of a scene, thereby giving a complete identification of the polarization state of light reflected from an object in the visible spectrum or emitted from an object in the long wave infrared spectrum. Such analysis allows the spatially varying two-dimensional state of polarization (SOP) to be determined. SOP analysis is a useful technique for object characterization and distinction. While the SOP from a scene examined over a sizeable band in the visible spectrum is dependent on a variety of factors, including wavelength ${ }^{10}$, solar angle, view angle, target angle, and the amount of skylight, the SOP from a scene in the LWIR is dependent only on the object's own characteristics such as temperature and shape. It is this driving force that makes broadband polarimetry more feasible in the LWIR than in the visible.

Current spectral analysis techniques used in remote sensing can suffer from a high degree of clutter, especially when operating over a small spectral band, resulting in object discrimination difficulties. ${ }^{1}$ It has already been recognized in the visible spectrum that greatly improved results from such systems, including general object recognition and characterization, can be achieved through the addition of polarization analysis. ${ }^{2}$ The implementation of polarimetric instruments into remote sensing systems for the purpose of identifying objects that are suggestive of nuclear proliferation activities is therefore a necessary step. Such an addition will provide improved discrimination algorithms that would be difficult to achieve from purely spectral techniques. Unfortunately, polarization signatures in the visible spectrum vary widely over the band and with illumination angles (solar angles of incidence). To ameliorate these issues, it is a logical extension to build and operate our system in the LWIR $(8-12 \mu \mathrm{m})$, where objects are self-luminous and the polarization signature of objects will not vary widely over this spectral band

\section{Table of Contents}

Section 1. Significance and Background Information ......................................... 3

Section 2. Identification of the Opportunity and Technical Approach........................... 3

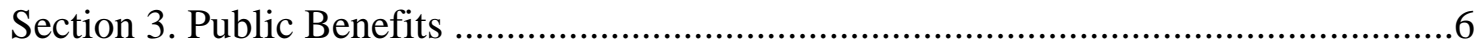

Section 4. Phase I Demonstrated Technical Feasibility..........................................

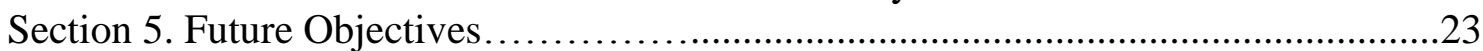

Section 6. Specific Future Work Plan...................................................................... 25

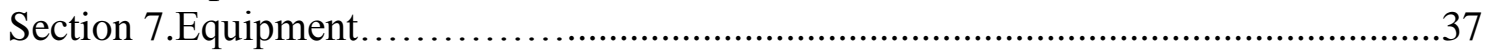

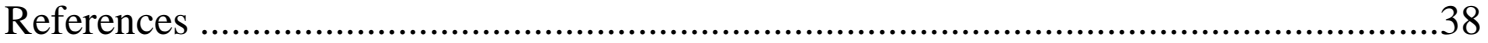




\section{Section 1. Significance and Background Information}

Previous remote sensing investigations have found that the more information available from the sensor, the more likely difficult detection and identification of objects of interest in the scene will be achieved. This is particularly true for treaty verification and nonproliferation applications where deliberate attempts at concealment and deception are made. Most remote sensing applications use spatial information from camera systems. More sophisticated sensor systems may also provide spectral information in addition to spatial data. In this project, we have developed a sensor that provides polarization information on the scene in addition to spatial information in order to provide additional capability for remote sensing information collection. The information provided by polarization sensors is particularly significant in remote sensing applications involving man made objects in natural backgrounds. This is particularly significant in the longwave infrared wavelength region where polarization data can provide insight into the source of radiation in the scene.

Current spectral analysis techniques used in remote sensing can suffer from a high degree of clutter, especially when operating over a small spectral band, resulting in object discrimination difficulties. ${ }^{1}$ It has already been recognized in the visible spectrum that greatly improved results from such systems, including general object recognition and characterization, can be achieved through the addition of polarization analysis. ${ }^{2}$ The implementation of polarimetric instruments into remote sensing systems for the purpose of identifying objects that are suggestive of nuclear proliferation activities is therefore a necessary step. Such an addition will provide improved discrimination algorithms that would be difficult to achieve from purely spectral techniques. Unfortunately, polarization signatures in the visible spectrum vary widely over the band and with illumination angles (solar angles of incidence). To ameliorate these issues, it is a logical extension to build and operate our system in the LWIR $(8-12 \mu \mathrm{m})$, where objects are self-luminous and the polarization signature of objects will not vary widely over this spectral band.

\section{Section 2. Identification of the Opportunity and Technical Approach}

Polarization images can provide a higher degree of contrast than an intensity image, providing the opportunity for dramatically improved object identification ${ }^{1}$. Furthermore, incorporation of a polarimeter allows for the potential to ascertain the Stokes vector elements of a scene, thereby giving a complete identification of the polarization state of light reflected from an object in the visible spectrum or emitted from an object in the long wave infrared spectrum. Such analysis allows the spatially varying two-dimensional state of polarization (SOP) to be determined.

SOP analysis is a useful technique for object characterization and distinction. While the SOP from a scene examined over a sizeable band in the visible spectrum is dependent on a variety of factors, including wavelength ${ }^{10}$, solar angle, view angle, target angle, and the amount of skylight, the SOP from a scene in the LWIR is dependent only on the object's 
own characteristics such as temperature and shape. It is this driving force that makes broadband polarimetry more feasible in the LWIR than in the visible. This is specifically demonstrated in figure 1.

SOP investigations that have already been performed point to a few general trends. Specifically, the generally smooth features of manmade objects will produce a significantly stronger polarization signature in comparison with the majority of natural objects. This tendency is indicative of the ability of polarimeters in remote sensing to supply information that purely spectral sensing would be unable to provide. Figure 1 provides photographs of a scene of interest with a polarization image and a color visible intensity image ${ }^{1}$.
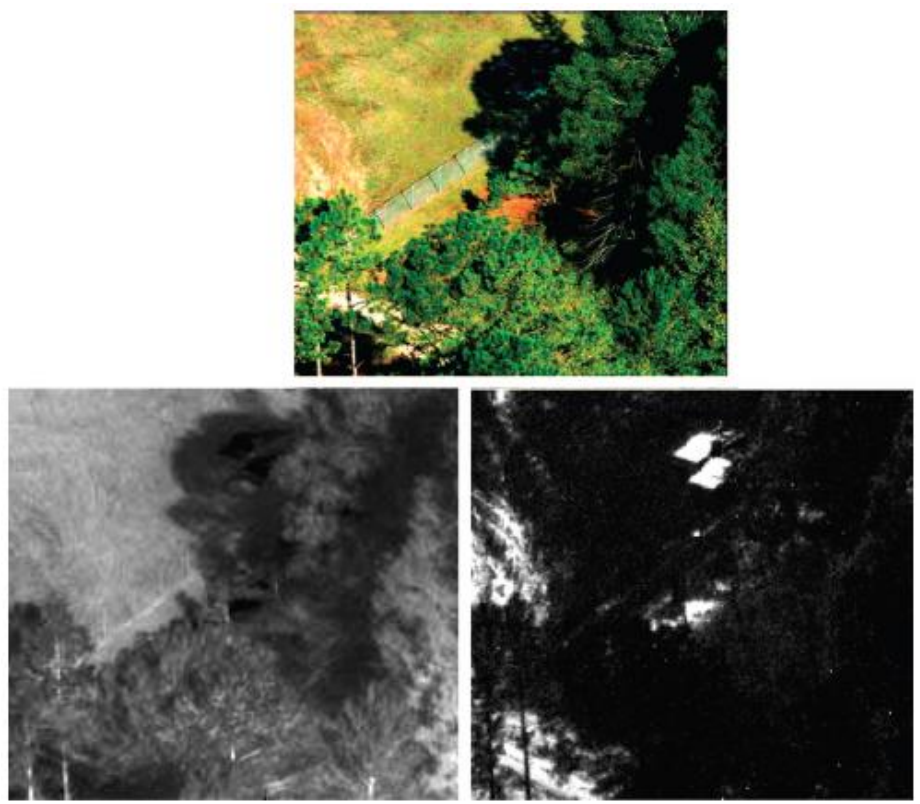

Figure 1 - Visible image (top) gives little indication of two trucks in the shadows. The trucks can be resolved a small degree better in the LWIR image (bottom left), however these trucks can be distinctly located in the LWIR polarization image (bottom right) ${ }^{1}$.

Most polarization sensors require rotating elements and multiple images to obtain polarization information. The sensor developed in this phase I STTR project has no moving parts, is compact, and provides complete polarization information in one frame time. .This project is for the design of a snapshot imaging Stokes polarimeter for remote sensing applications in the LWIR wavelength band. In order to accomplish the desired snapshot capability of the system, image shearing based on incident object polarization is required. Each sheared image is then recombined at the image plane and the resulting fringe pattern is analyzed to obtain the polarization content of the image through spatial frequency filtering analysis. The shearing mechanism is executed through the use of Sagnac interferometers and retarders.

The polarization content of the image is obtained through interferometric analysis. Because of this, coherence is a large issue in determining the quality of the fringe patterns. Only temporal coherence will have an appreciable affect on the fringe visibility, however. Using standard imaging optics, fringe visibility drops to $50 \%$ over a miniscule 
bandwidth. Hence, while using only standard refractive optics, only a narrow band instrument could be constructed. To ameliorate this issue we will use an achromatic Fourier transforming lens system (AFT) to attempt to correct for the wavelength dependence of the fringe visibility and drastically improve the bandwidth.

To accomplish this, we designed a novel, compact, broadband, thermal infrared snapshot complete imaging Stokes polarimeter as part of a remote sensing system. Through the design of this passive broadband instrument, signal to clutter is expected to be vastly superior when compared to narrow band analysis, and temporal artifacts associated with other polarimeters are eliminated. The designed system, shown in figure 2, consists of a reflective Ritchey-Chretien telescope that produces an intermediate image of the scene followed by a modified white-light optical correlator ${ }^{6}$ with shearing Sagnac interferometers providing the aperture plane transmittance function.

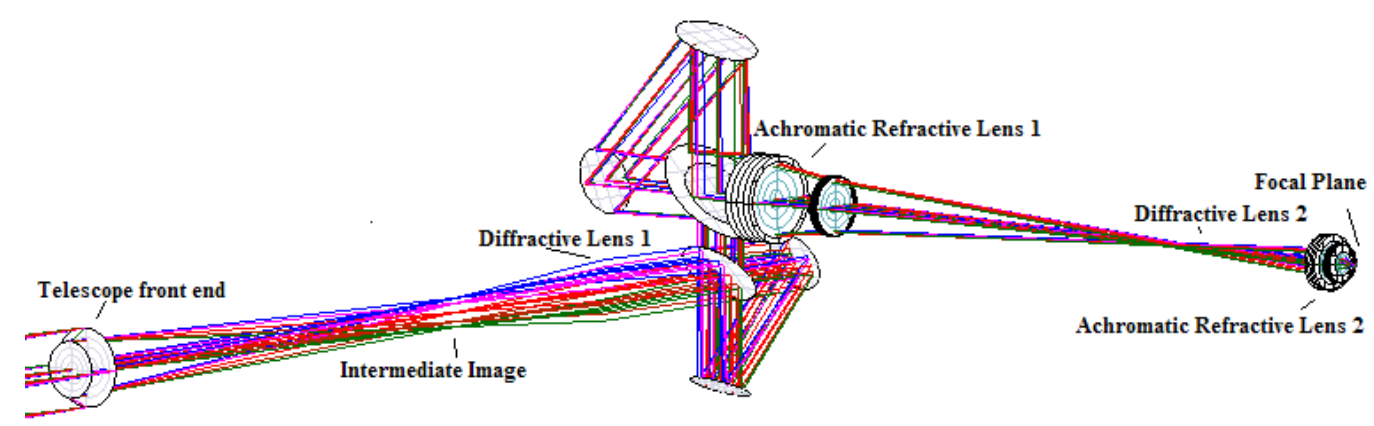

Figure $2-\mathrm{CodeV}$ 3D model of the designed system. Broadband LWIR radiation from the scene is incident on the telescopic front end is eventually achromatically imaged to the focal plane.

Essentially, this system is a modified combination of an Achromatic Fourier Transform lens system (AFT) and a Sagnac shearing interferometer snapshot imaging polarimeter, which is simply a Savart plate snapshot imaging polarimeter with Sagnac shearing interferometers substituted for Savart plates. This substitution is necessary because materials for Savart plates in the LWIR are generally unavailable. Savart plate imaging polarimeter technology has already been established for narrow bandwidths and has been demonstrated to provide complete polarization information with no need for moving parts. This is accomplished through the use of carrier fringes and spatial frequency filtering. ${ }^{3}$ These fringes are formed on the back focal plane of the polarimeter. This system was designed in this Phase 1, and theoretically allows for broadband polarization measurements, which have not yet been achieved with this method. Transforming this narrow band polarimeter into a broadband instrument makes it ideal for remote sensing applications. This system can also be extended into other spectral bands, as long as the requisite materials are available and the polarization signature is not strongly spectrally dependent.

The system can be made into a more compact 2D layout for ease of construction if a silvered image rotation prism is used immediately following the first Sagnac interferometer, by rotating the orientation of the transmitted radiation by 90 degrees. 
There may be minor polarization effects in doing this, but they can be easily calibrated out. This folded system is shown below,

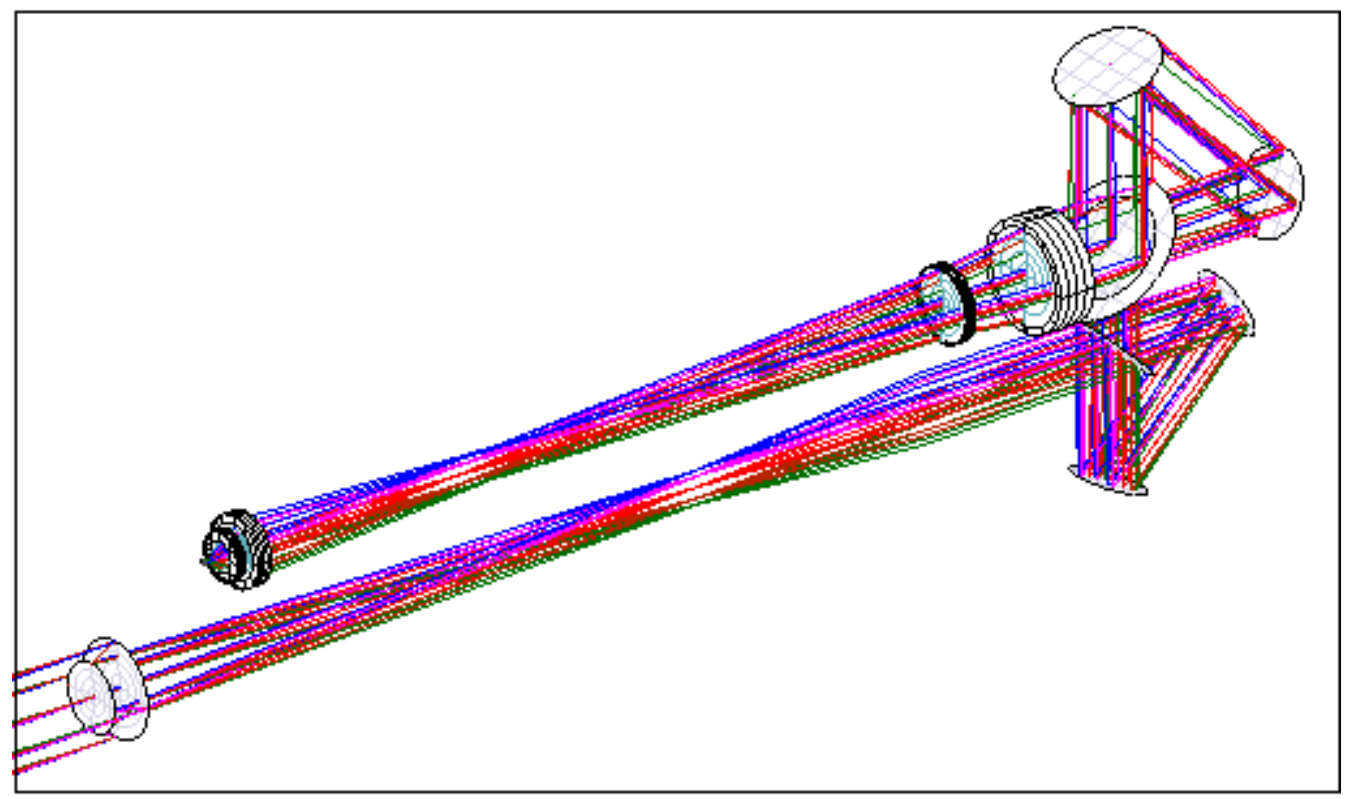

Figure 3 - Folded system design from figure 2.

\section{Section 3. Anticipated Public Benefits}

We expect the public to benefit from the polarimetric imaging techniques and algorithms to be developed, which have the potential to increase object detection through the reduction of clutter. This technology for material surface characterization may be used for the identification, classification, and mapping of,

1. Processes suggestive of nuclear proliferation, weapons of mass destruction, or similar activities. ${ }^{4}$

2. A variety of topographical regions on the Earth's surface for determination of composition, i.e. the amount of vegetation, watershed, rock versus soil, etc.

3. Active and potential regions of change on the Earth, such as landslides and slope stability. ${ }^{2}$

4. Cancer and other diseases in the body. ${ }^{5}$

These examples are a few of the many potential benefits. Pursuit of any or all of these applications has potential to lead to the production of a marketable product.

Based on these applications, there are a number of commercial groups that would find interest in the development of this technology. Topographical and slope stability mapping and analysis are of interest to the geology industry, as well as to the construction industry for identification of suitable erection locations and other work. Even the logging industry 
may be interested in an isolated means of locating areas for possible harvesting. Polarization imaging studies have the potential for identifying such areas remotely, which can then be assessed directly with more precision. The medical industry would also benefit from the development of this technology as a means of improving in-vivo cancer detection; work in this area has already demonstrated the usefulness of imaging polarimetry for this application. ${ }^{5}$

In addition, several divisions within the Federal Government beyond the Department of Energy (DOE) may profit from the success of this technology. The Department of Defense (DOD) may find use of polarimetric measurements for real time analysis and differentiation of battlefield targets. The US Geological Survey (USGS), the Bureau of Land Management (BLM), the Department of Transportation (DOT) and the National Oceanic and Atmospheric Administration (NOAA) may all have interest in a new means for identifying regions and areas of change on the Earth's surface for water and land management.

\section{Section 4. Phase I Demonstrated Technical Feasibility}

The objective of this Phase 1 SBIR effort was to develop a detailed design for a broadband snapshot imaging Stokes polarimeter capable of determining all four Stokes vectors and operates in the long wave infrared (LWIR) spectral region. Three major activities were required to achieve this overall objective. First, we collected data on potential components and materials. Second, we developed a detailed design for the entire system. Third, we used theoretical models to study the system performance under specific tolerances. The specific technical objectives and issues they addressed concerning the Phase I effort were as follows:

Technical Objective A. Collect Data on Potential Components and Materials

The proposed sensor design has not been implemented in this configuration. Therefore, we had to carefully select materials and components based upon their detailed material properties and characteristics. We must obtain or measure certain material and behavioral properties which are necessary to ensure proper sensor performance. This will ensure satisfactory calibration and sensor performance when the sensor is constructed. During the Phase I effort, this forced us to use a Sagnac interferometer instead of a Savart plate, because of a lack of feasible Savart plate materials in the LWIR.

Technical Objective B. Develop a Detailed System Design

1) Construct an achromatic Fourier transforming lens (AFT) model to determine viability of this technique for incoherent interferometric testing, specifically for remote sensing. This objective addressed:

i) How the use of spectrally broad, spatially varying, distant objects as illumination sources affected results in the detection plane.

ii) How much of the LWIR spectrum could be utilized while still producing uniform lateral magnification. 
2) Develop a complete system model, which includes both the AFT and the Sagnac interferometer polarimeter which will provide a detailed analysis and complete indication of project feasibility. A variety of factors were addressed with this objective.

i) System performance as a pure achromatic imager.

ii) Quantitative values for expected field of view and resolution.

iii) Develop system tolerances and specifications using ray-trace software.

\subsection{Technical Objective A Research}

Polarization images can provide a higher degree of contrast than an intensity image, providing the opportunity for dramatically improved object identification ${ }^{1}$. Furthermore, incorporation of a polarimeter allows for the potential to ascertain the Stokes vector elements of a scene, thereby giving a complete identification of the polarization state of light reflected from an object in the visible spectrum or emitted from an object in the long wave infrared spectrum. Such analysis allows the spatially varying two-dimensional state of polarization (SOP) to be determined.

Current techniques for imaging polarimetry include the use of a rotating retarder followed by an analyzer, which are placed in front of the detection system. Through a series of measurements, the complete Stokes vector of the scene can be determined. This method imposes several difficulties. Most obviously, rotating parts can lead to vibrational and mechanical problems. Dynamic scenes can also create difficulties when methods requiring a series of measurements are used. In addition, this technique suffers from oversampling and difficulties with spatial synchronization ${ }^{1,3}$.

As a means of overcoming some of the problems with currently employed approaches, our intended design takes advantage of other well-documented technologies, which to our knowledge have not been combined for the purpose of imaging polarimetry. Specifically, we designed a system in the LWIR that would work like a Savart plate (SP) imaging polarimeter $^{3,8}$ in conjunction with an achromatic Fourier transforming lens ${ }^{6}$ (AFT) in order to provide complete polarization analysis of a scene over the entire LWIR (8-12 $\mu \mathrm{m})$. The system will provide higher fidelity polarization data due to its snapshot nature.

The SP imaging polarimeter uses Savart plates to shear incident radiation based on its polarization content. In the infrared, the Savart plates are essentially unavailable. Instead of using the Savart plates for shearing the incident beam based on polarization, Sagnac interferometers can be used instead. These interferometers can accomplish the same degree of shearing in the infrared as the Savart plates can in the visible. An example of a shearing Sagnac interferometer is given in the following figure: 


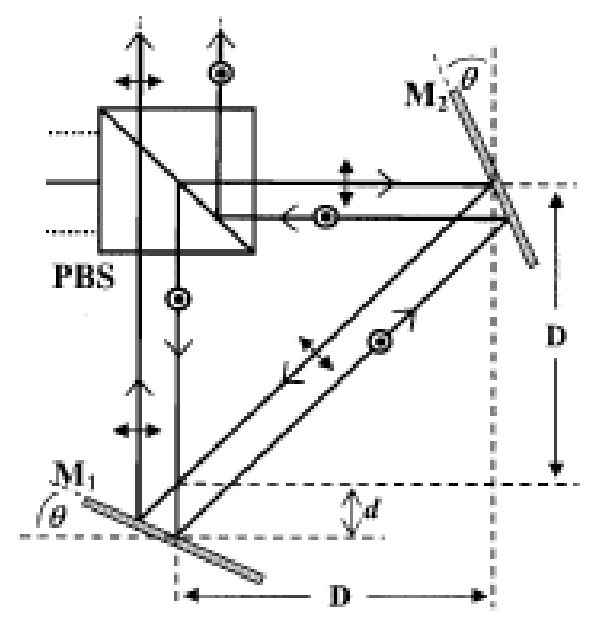

Figure 4 - Shearing Sagnac Interferometer (SSI) ${ }^{7}$

The Sagnac interferometer shears the incident beam based on its polarization. The amount of introduced shear is based solely on the parameter, $d$, in figure 4 . We modeled such an interferometer in the Thermal infrared in Code V. However, instead of the actual beam splitter cube that will be used, we modeled the polarization beam splitter as a tilted flat mirror and a polarizer for one polarization and just a single polarizer for the orthogonal polarization. Using $\mathrm{d}=10 \mathrm{~mm}$, the following systems were produced:
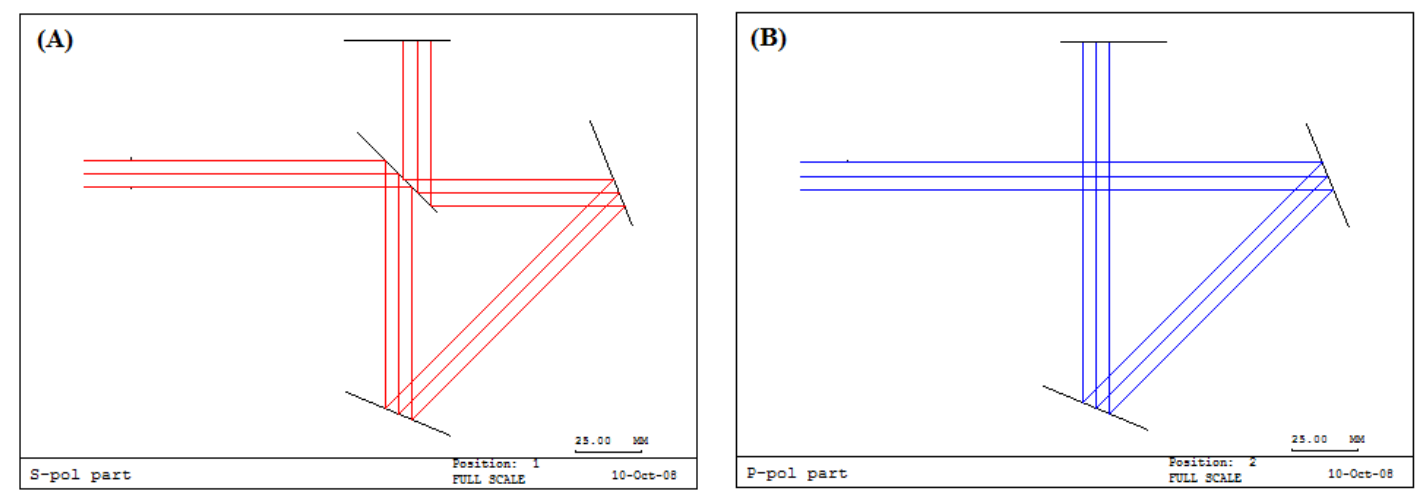

Figure 5 - (A) $\mathrm{S}$ - polarized component as it is sheared through the interferometer; (B) $\mathrm{P}$ - polarized component as it is sheared through the interferometer.

When they are superimposed, you will observe that the chief rays are displaced with respect to one another on the focal plane, which is the horizontal plane at the top of figure 5. The accompanying display in figure 6 is the beam footprint at the focal plane as seen in the $\mathrm{X}-\mathrm{Y}$ plane. The lens design drawings are viewed in the $\mathrm{Y}-\mathrm{Z}$ plane. 


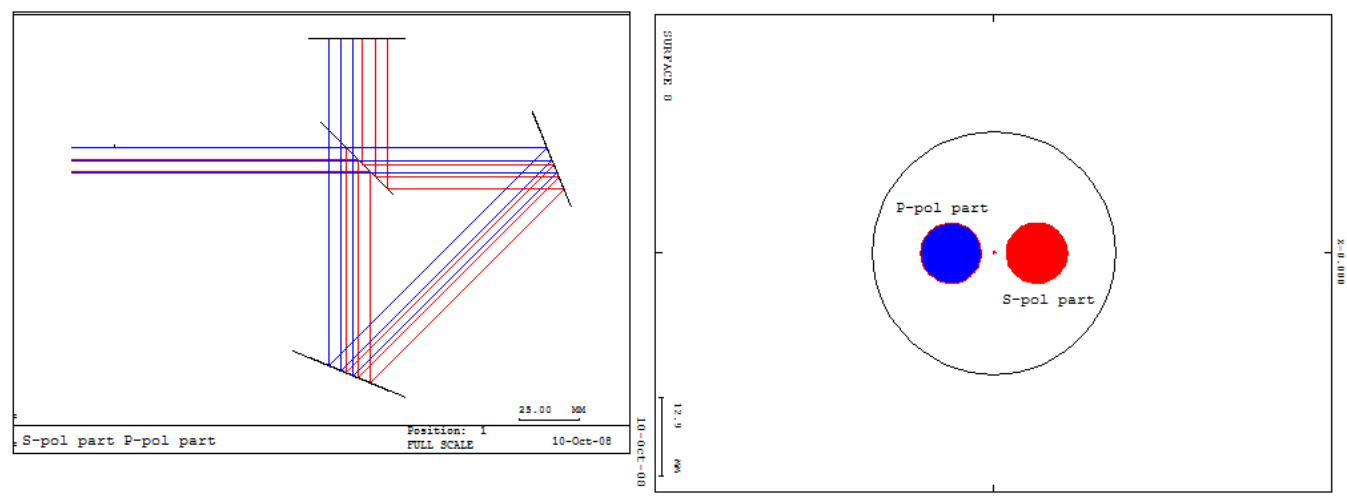

Figure 6 - (A) Sagnac interferometer shearing S and P polarizations. (B) Beam footprint at the focal plane.

The polarization dependant shearing is readily observed in figure 6 . We intentionally made the shear more severe than we would design in the actual interferometer to demonstrate the ability to shear the incident beam. In the SP imaging polarimeter, two SP's are used. The same is true in the LWIR adaptation; two Sagnac shearing interferometers are used. The initial beam is sheared into four independent beams based on its polarization. This is shown in figure 7.

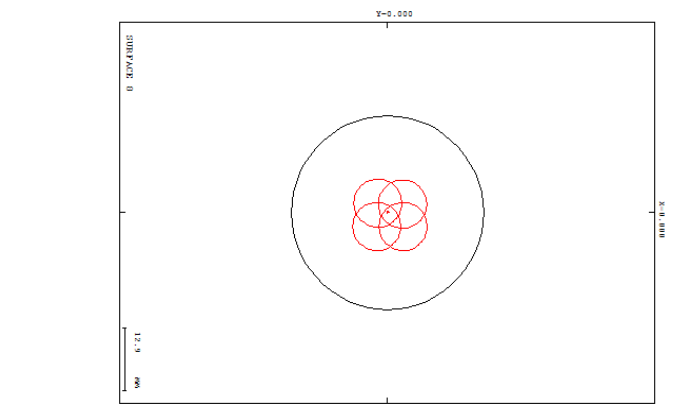

Figure 7 - Output of coupled Sagnac interferometers.

The polarizing beam splitters are possible to obtain in the LWIR, but do have high associated costs. Instead of using individual polarizing beam splitters, a non-polarizing $50 / 50$ beam splitter followed by a polarizer, half-wave retarder, and a second polarizer whose axis is orthogonal to the first can simulate a polarizing beam splitter. Using this configuration is not light efficient, however.

\subsection{Technical Objective B Research}

Luo and Oka have documented the use of SP technology for the determination of the two-dimensional SOP of visible light for a narrow spectral band. ${ }^{8}$ Their snapshot system, as diagrammed in figure 8 , does not require the use of rotating or otherwise dynamic components, but instead takes advantage of spatial carrier fringes in order to provide a complete polarization analysis of the object of interest, using channeled polarimetry ${ }^{3}$. 


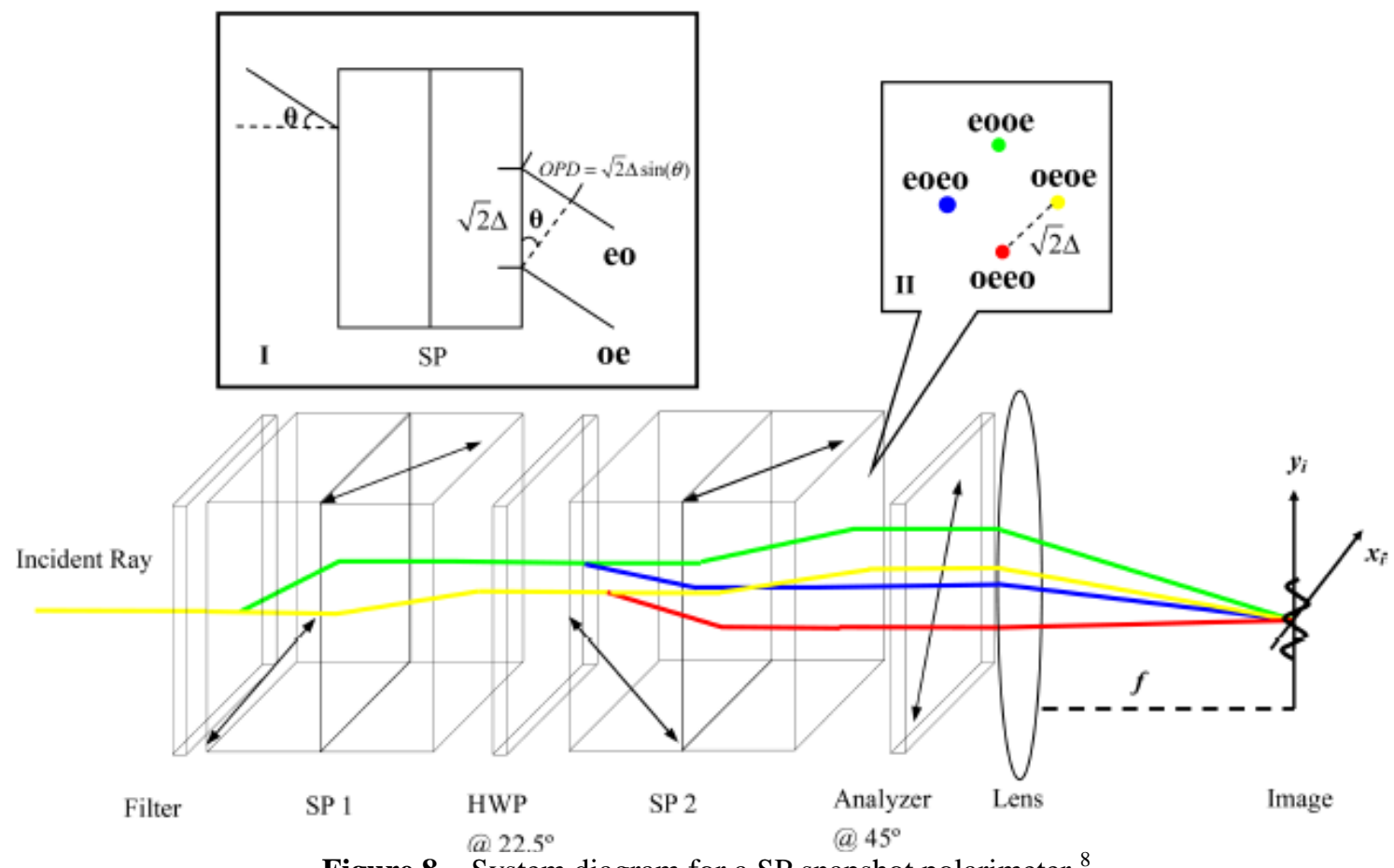

Figure 8 - System diagram for a SP snapshot polarimeter. ${ }^{8}$

Using a pair of SP's, which have been rotated at 90 degrees with respect to one another, light incident on the system is sheared to create four separate beams. After passing through an analyzer, these beams are recombined, resulting in interference fringes containing the complete State of Polarization (SOP) information on the image plane. Quantitatively, the irradiance on the image plane is given by ${ }^{8}$,

$$
\begin{aligned}
I\left(x_{i}, y_{i}\right)= & \frac{1}{2} S_{0}+\frac{1}{2} S_{1} \cdot \cos \left(2 \pi \Omega\left(x_{i}+y_{i}\right)\right) \\
& +\frac{1}{4}\left|S_{23}\right| \cdot \cos \left[2 \pi(2 \Omega) x_{i}-\arg \left(S_{23}\right)\right] \\
& -\frac{1}{4}\left|S_{23}\right| i \cdot \cos \left[2 \pi(2 \Omega) y_{i}+\arg \left(S_{23}\right)\right] \\
S_{23}= & S_{2}+i S_{3}, \quad \Omega=\frac{\Delta}{\lambda f} .
\end{aligned}
$$

where $S_{0}, S_{1}, S_{2}$, and $S_{3}$ are all of the Stokes vector components that describe the complete polarization content of radiation.

This technique has been verified experimentally ${ }^{8}$ using commercially available components. Figure 9 demonstrates the feasibility of such a narrow band instrument: 


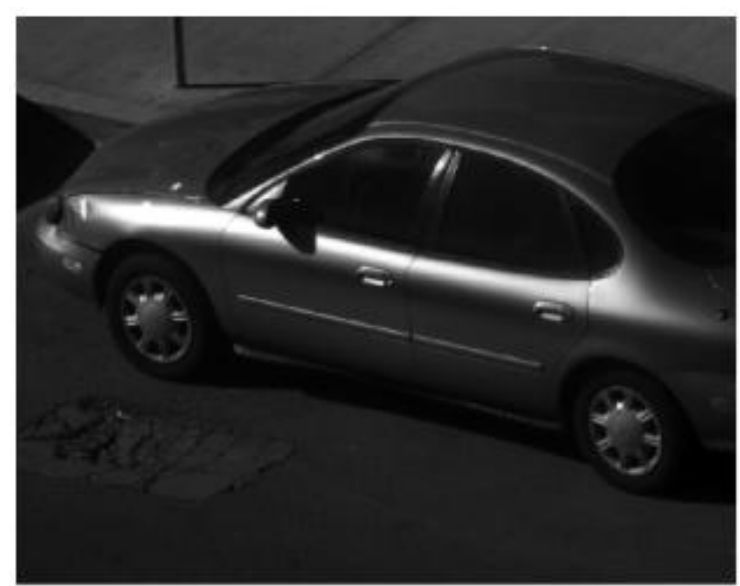

(a)

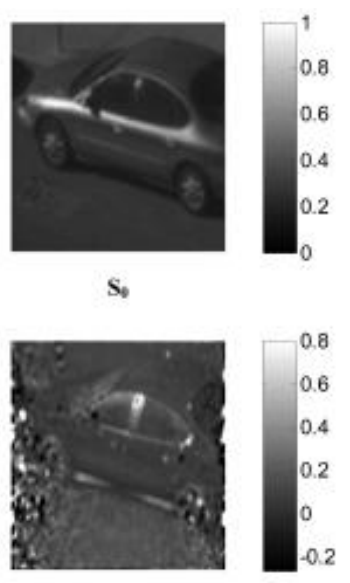

$\mathbf{S}_{2}$

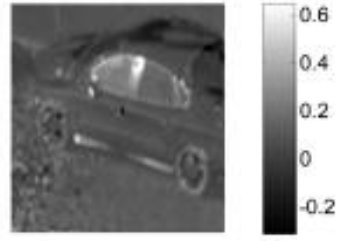

S)

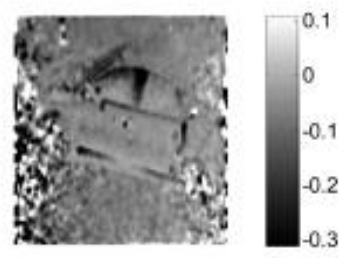

$\mathbf{S}_{3}$

(b)

Figure 9 - (a) Raw image of a scene of interest; (b) Reconstructed Stokes images that describe the complete polarization content of the scene of interest using the SP snapshot polarimeter.

It is imperative that any model that is based on our design give the same form as that seen in equation (1). The entire designed system consists of multiple refractive, reflective, diffractive, and polarization elements. In sequence, an incoming ray, after the intermediate image will encounter a diffractive optical element, followed by a Sagnac shearing interferometer that will shear in the y-direction, next it will meet with a halfwave plate oriented at 22.5 degrees, after the wave plate, the ray goes through another Sagnac shearing interferometer that will shear the split ray in the x-direction. After the final Sagnac interferometer, the ray traverses through a linear polarizer oriented at 45 degrees. At this point there are four individual sheared beams in the same polarization state. These beams then go through the imaging system that consists of two air-spaced achromatic refractive lenses and a diffractive lens, not in that order. After recombination at the image plane, the four beams interfere based on the polarization of the objects in the scene of interest. A 3-dimensional representation of the system was given in figure 2 .

A Jones matrix model is appropriate for studying the monochromatic behavior of the designed system. The Jones matrix model only concerns itself with polarization sensitive elements. These elements include Sagnac interferometers, polarizers, waveplates, and mirrors. The imaging elements are modeled as perfect elements in the Jones matrix model and as such have no effect on the polarization content of the final electric field. Also, since there are even numbers of reflections in the system before each polarization element, we need not include mirror reflection matrices in the model.

The Sagnac interferometers are modeled as phase shifting elements. The optical path difference (OPD) between orthogonally polarized rays entering the interferometer is only a function of incident angle and d, mirror displacement in each Sagnac. A schematic of the Sagnac was given in figure 4.

The OPD is given as $\frac{\sqrt{2}}{2} d \sin \theta_{x, y i n c}$ for each polarization. With the Sagnac interferometer that shears the beam in the y-direction, the S-polarized component of the 
E-field has positive OPD and the P-polarized component has negative OPD. Therefore, the Jones matrix for the $y$-shearing Sagnac is given as

$$
S_{y}=\left(\begin{array}{cc}
\exp \left[i \phi_{A}\right] & 0 \\
0 & \exp \left[i \phi_{B}\right]
\end{array}\right)
$$

where,

$$
\begin{aligned}
& \phi_{A}=\left(\frac{2 \pi}{\lambda}\right) \frac{\sqrt{2}}{2} d \sin \theta_{y} \approx\left(\frac{2 \pi}{\lambda f}\right) \frac{\sqrt{2}}{2} d y \\
& \phi_{B}=-\left(\frac{2 \pi}{\lambda}\right) \frac{\sqrt{2}}{2} d \sin \theta_{y} \approx-\left(\frac{2 \pi}{\lambda f}\right) \frac{\sqrt{2}}{2} d y
\end{aligned}
$$

Similarly,

$$
S_{x}=\left(\begin{array}{cc}
\exp \left[i \phi_{C}\right] & 0 \\
0 & \exp \left[i \phi_{D}\right]
\end{array}\right)
$$

where,

$$
\begin{aligned}
& \phi_{C}=\left(\frac{2 \pi}{\lambda}\right) \frac{\sqrt{2}}{2} d \sin \theta_{x} \approx\left(\frac{2 \pi}{\lambda f}\right) \frac{\sqrt{2}}{2} d x \\
& \phi_{D}=-\left(\frac{2 \pi}{\lambda}\right) \frac{\sqrt{2}}{2} d \sin \theta_{x} \approx-\left(\frac{2 \pi}{\lambda f}\right) \frac{\sqrt{2}}{2} d x
\end{aligned}
$$

The complete Jones matrix output will be as follows:

$$
\vec{E}_{\text {out }}=\left(\begin{array}{cc}
1 / 2 & 1 / 2 \\
1 / 2 & 1 / 2
\end{array}\right)\left(\begin{array}{cc}
\exp \left[i \phi_{C}\right] & 0 \\
0 & \exp \left[i \phi_{D}\right]
\end{array}\right)\left(\begin{array}{cc}
\frac{\sqrt{2}}{2} & \frac{\sqrt{2}}{2} \\
\frac{\sqrt{2}}{2} & -\frac{\sqrt{2}}{2}
\end{array}\right)\left(\begin{array}{cc}
\exp \left[i \phi_{A}\right] & 0 \\
0 & \exp \left[i \phi_{B}\right]
\end{array}\right)\left(\begin{array}{l}
E_{x} \\
E_{y}
\end{array}\right)
$$

completing the matrix algebra,

$$
\vec{E}_{\text {out }}=\frac{\sqrt{2}}{2}\left(\begin{array}{l}
\frac{E_{x}}{2}\left(\exp \left[i\left(\phi_{C}+\phi_{A}\right)\right]+\exp \left[i\left(\phi_{D}+\phi_{A}\right)\right]\right)+\frac{E_{y}}{2}\left(\exp \left[i\left(\phi_{C}+\phi_{B}\right)\right]-\exp \left[i\left(\phi_{D}+\phi_{B}\right)\right]\right) \\
\left.\frac{E_{x}}{2}\left(\exp \left[i\left(\phi_{C}+\phi_{A}\right)\right]+\exp \left[i\left(\phi_{D}+\phi_{A}\right)\right]\right)+\frac{E_{y}}{2}\left(\exp \left[i\left(\phi_{C}+\phi_{B}\right)\right]-\exp \left[i\left(\phi_{D}+\phi_{B}\right)\right]\right)\right)
\end{array}\right)
$$

What we observe on the image plane is the irradiance pattern of the incident beam and not the electric field. Consequently, we must calculate the irradiance. The irradiance on the image plane is calculated as follows:

$$
I=\left\langle E^{*} E\right\rangle=\left\langle\left|\frac{E_{y}}{2} \exp \left[i \phi_{1}\right]-\frac{E_{y}}{2} \exp \left[i \phi_{2}\right]+\frac{E_{x}}{2} \exp \left[i \phi_{3}\right]+\frac{E_{x}}{2} \exp \left[i \phi_{4}\right]\right|^{2}\right\rangle
$$


where,

$$
\begin{aligned}
& \phi_{1}=\left(\frac{2 \pi}{\lambda f}\right)\left(\frac{\sqrt{2}}{2} d\right)(x-y) \\
& \phi_{2}=\left(\frac{2 \pi}{\lambda f}\right)\left(\frac{\sqrt{2}}{2} d\right)(-x-y) \\
& \phi_{3}=\left(\frac{2 \pi}{\lambda f}\right)\left(\frac{\sqrt{2}}{2} d\right)(x+y) \\
& \phi_{4}=\left(\frac{2 \pi}{\lambda f}\right)\left(\frac{\sqrt{2}}{2} d\right)(-x+y)
\end{aligned}
$$

Using the following relations ${ }^{8}$, the total irradiance pattern can be calculated as a function of Stokes vector components:

$$
\begin{aligned}
& \left\langle\left|E_{x}\right|^{2}\right\rangle+\left\langle\left|E_{y}\right|^{2}\right\rangle=S_{0}, \quad\left\langle\left|E_{x}\right|^{2}\right\rangle=\frac{1}{2}\left(S_{0}+S_{1}\right), \\
& \left\langle\left|E_{y}\right|^{2}\right\rangle=\frac{1}{2}\left(S_{0}-S_{1}\right), \quad\left\langle E_{x}^{*} E_{y}\right\rangle=\frac{1}{2}\left(S_{2}+i S_{3}\right) .
\end{aligned}
$$

The total irradiance pattern is calculated:

$$
I=\frac{1}{4}\left\{\begin{array}{l}
2 S_{0}-\left[\left(S_{0}-S_{1}\right)\left(\exp \left[i\left(\phi_{2}-\phi_{1}\right)\right]+\exp \left[-i\left(\phi_{2}-\phi_{1}\right)\right]\right)\right]+ \\
{\left[\left(S_{0}+S_{1}\right)\left(\exp \left[i\left(\phi_{4}-\phi_{3}\right)\right]+\exp \left[-i\left(\phi_{4}-\phi_{3}\right)\right]\right)\right]+} \\
{\left[\left(S_{2}+i S_{3}\right) \exp \left[i\left(\phi_{3}-\phi_{1}\right)\right]+\left(S_{2}-i S_{3}\right) \exp \left[i\left(\phi_{3}-\phi_{1}\right)\right]\right]-} \\
{\left[\left(S_{2}+i S_{3}\right) \exp \left[i\left(\phi_{4}-\phi_{2}\right)\right]+\left(S_{2}-i S_{3}\right) \exp \left[i\left(\phi_{4}-\phi_{2}\right)\right]\right]+} \\
\left.\left[\left(S_{2}\right)\right]+\left(S_{2}-i S_{3}\right) \exp \left[i\left(\phi_{4}-\phi_{1}\right)\right]\right]- \\
\left.\operatorname{loxp}\left[i\left(\phi_{3}-\phi_{2}\right)\right]+\left(S_{2}-i S_{3}\right) \exp \left[i\left(\phi_{3}-\phi_{2}\right)\right]\right]
\end{array}\right\}
$$

rearranging terms,

$$
I=\left\{\begin{array}{l}
\frac{1}{2} S_{0}+\frac{1}{2} S_{1} \cos \left(\frac{2 \pi \sqrt{2} d}{\lambda f} x\right)+ \\
\frac{1}{4}\left|S_{23}\right| \cos \left(\frac{2 \pi \sqrt{2} d}{\lambda f}(x-y)+\Gamma\right)- \\
\frac{1}{4}\left|S_{23}\right| \cos \left(\frac{2 \pi \sqrt{2} d}{\lambda f}(x+y)-\Gamma\right)
\end{array}\right\}
$$


where,

$$
\begin{aligned}
& S_{2} \pm i S_{3}=\left|S_{23}\right| e^{i \Gamma}, \\
& \left|S_{23}\right|=\sqrt{S_{2}^{2}+S_{3}^{2}}, \Gamma=\tan ^{-1}\left[\frac{S_{3}}{S_{2}}\right]
\end{aligned}
$$

Equation 10 is a very important result. It shows that all of the Stokes parameters are modulated by individual carrier frequencies. This allows us to simultaneously image and obtain polarization information in the scene in a snapshot via inverse Fourier transform using Sagnac shearing interferometers in place of Savart plates. This also agrees heavily with equation 1 , a result that was proven experimentally by Luo and Oka ${ }^{8}$.

Now that it has been established that the narrow band visible SP imaging polarimeter can be adapted to the LWIR through the use of Sagnac shearing interferometers (SSI) instead of Savart plates, we need to determine how to make the system broadband.

Initially, it was thought that we could simply present the first SSI as the front end of the system, but Fresnel diffraction is strongly dispersive, and as such would de-achromatize any results, if we were using this system in a remote sensing capacity. Essentially, it is wavelength dependent diffraction from the objects themselves that would cause issues in this attempt to make the instrument broadband. The designed system that solved the issue of wavelength dependent diffraction from the objects themselves consists of presenting an intermediate image of the scene through the use of an all-reflective Ritchey-Chretien telescope. Following the image downstream, a first diffractive lens is placed immediately before the Sagnac shearing interferometers, after the first Sagnac shearing interferometer, a half-wave retarder is placed. After the half-wave retarder, we place the second Sagnac shearing interferometer followed by a polarizer and an achromatic refractive lens, and following the refractive lens we place a second diffractive lens. Finally, a second refractive lens is added following the entire system. The entire system is shown in figure 2 , but is again presented below.

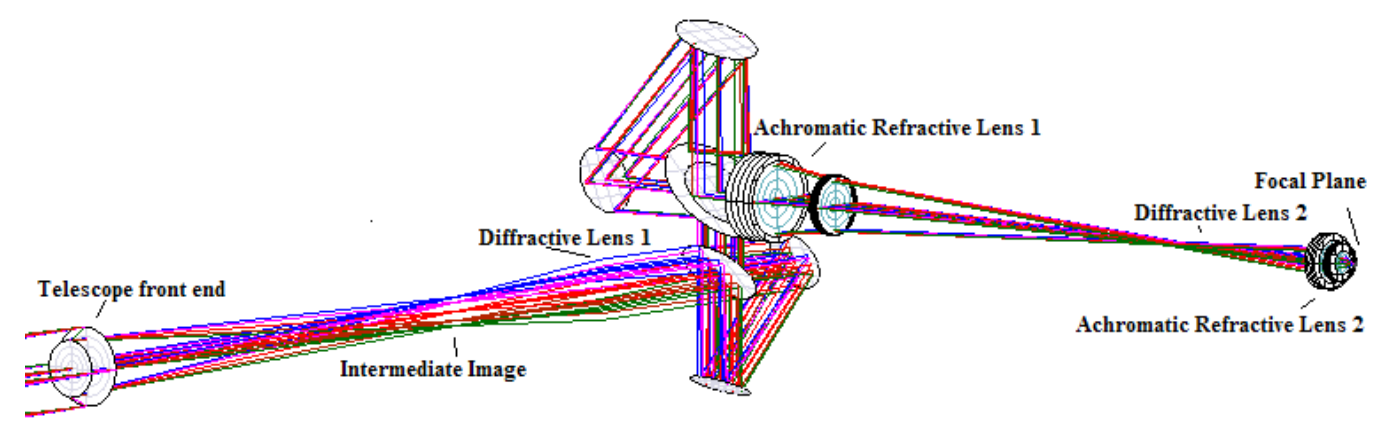

Figure 2(revisited) - CodeV 3D model of the designed system. Broadband LWIR radiation from the scene is incident on the telescopic front end is eventually achromatically imaged to the focal plane. 
This system attains an achromatic PSF by intelligent positioning of each element of the system. This system is essentially a three-lens optical correlator with a fourth lens added to image a virtual output plane into a sensible object space location. This is our achromatic Fourier transforming lens system (AFT).

A simple schematic of the system, with the exception of the last refractive lens is given:

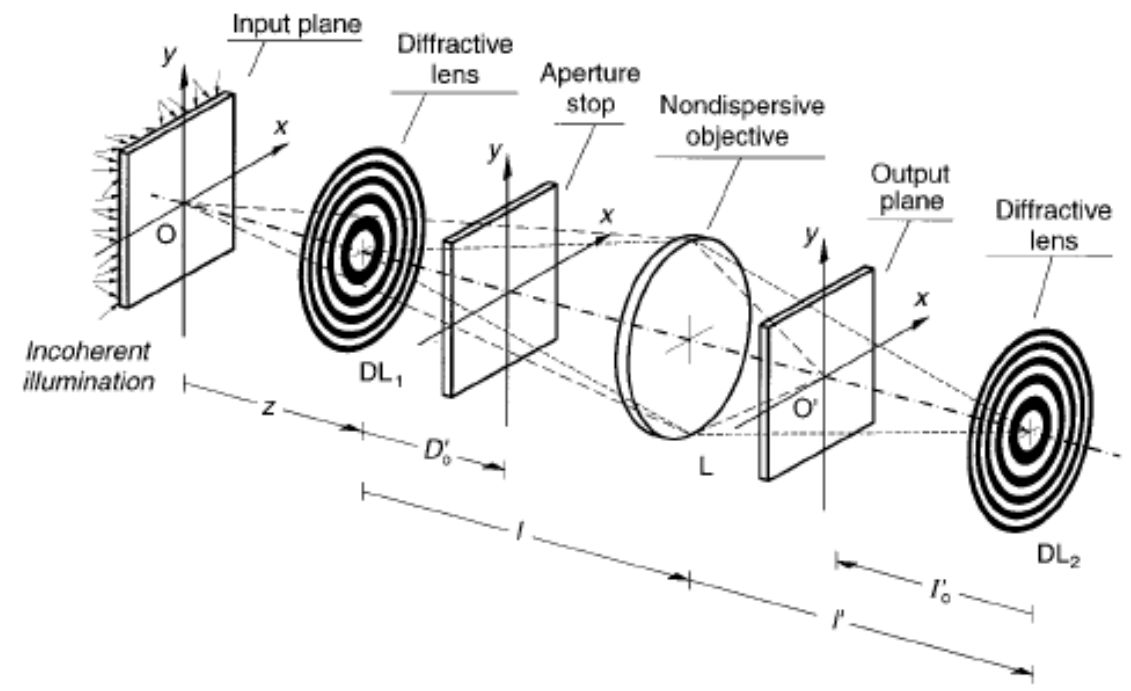

Figure 10 - Three-lens scale-tunable optical correlator. ${ }^{6}$

The transmission function (matrix multiplying the incident E-field in equation (6) of the Sagnac interferometer systems is essentially the mask function that is placed at the aperture stop location. This function is multiplied by the Fourier transform of the input object which would be the intermediate image of our scene of interest. The achromatic Fourier transform of this combination is our desired output and it is given in the output plane, which is achromatically imaged into a real image in object space through the use of a second achromatic refractive lens not shown in figure 10.

The constraints for the positioning of each of these elements are given by Gladys Minguez-Vega, et al ${ }^{6}$. Through the use of ray matrices and wave optics, they determined the following constraints ${ }^{6}$ :

$$
\begin{gathered}
\frac{1}{l}+\frac{1}{l^{\prime}}=\frac{1}{f}, Z_{0}{ }^{\prime}=-M^{2} Z_{0}, \quad I_{0}{ }^{\prime}=-M M_{0} z, \\
D_{0}{ }^{\prime}=\frac{z Z_{0}}{2 z-Z_{0}}, \quad \text { and } z>Z_{0} / 2
\end{gathered}
$$

where $\mathrm{f}$ is the focal length of the first refractive lens, $M=\frac{-l^{\prime}}{l}, M_{0}=\frac{-f}{z+l-f}$, which are the lateral magnification between the planes containing the diffractive lenses and between the input and output planes respectively. The effective focal lengths of the diffractive 
lenses are $Z_{1}=\frac{Z_{0} \sigma}{\sigma_{0}}$, and $Z_{2}=\frac{Z_{0}{ }^{\prime} \sigma}{\sigma_{0}}$, respectively, where $Z_{0}$ and $Z_{0}$ ' are the focal lengths at the design wavenumber, $10 \mu \mathrm{m}$.

To evaluate the coherent point spread function (PSF), $h(x, y, \sigma)$, where $\sigma$ is the wave number, from the aperture stop with transmittance $t(x, y)$ to the output plane we consider a broadband point source a distance $\mathrm{z}$ away from the first diffractive lens with focal length $Z_{1}$. The aperture stop is located a distance, $D_{o}$ ' away from the first diffractive lens. In this configuration, the diffracting aperture is illuminated with a spatial continuum of point sources, each radiating at a different wavelength, along the optical axis. This is a result of the imaging of the broadband point source through the first diffractive lens ${ }^{6}$. The distance each of these point sources focus away from the aperture stop is given by

$$
s(\sigma)=D_{o}^{\prime}-\left[\frac{\sigma_{0}}{Z_{0} \sigma}-\frac{1}{z}\right]^{-1}
$$

Using ray matrices, the $\mathrm{ABCD}$ matrix characterizing the optical system from the aperture stop to the output plane, we find ${ }^{6}$,

$$
\begin{aligned}
{\left[\begin{array}{ll}
A & B \\
C & D
\end{array}\right]=} & {\left[\begin{array}{ll}
1 & I_{0}^{\prime} \\
0 & 1
\end{array}\right]\left[\begin{array}{cc}
1 & 0 \\
-1 / Z_{2} & 1
\end{array}\right]\left[\begin{array}{cc}
1 & l^{\prime} \\
0 & 1
\end{array}\right]\left[\begin{array}{cc}
1 & 0 \\
-1 / f & 1
\end{array}\right] } \\
& \times\left[\begin{array}{ccc}
1 & l-D_{0}^{\prime} \\
0 & 1
\end{array}\right]\left[\begin{array}{cc}
1 & 0 \\
1 / s(\sigma) & 1
\end{array}\right]
\end{aligned}
$$

When using ray matrices, the complex field distribution in an output plane is given by,

$$
\begin{aligned}
& U_{\text {out }}(x, y ; \sigma) \\
& =\exp \left[i \pi \sigma \frac{D}{B}\left(x^{2}+y^{2}\right)\right] \\
& \quad \times \int_{-\infty}^{\infty} \int U_{\text {in }}\left(x^{\prime}, y^{\prime} ; \sigma\right) \exp \left[i \pi \sigma \frac{A}{B}\left(x^{\prime 2}+y^{\prime 2}\right)\right] \\
& \quad \times \exp \left[-\frac{i 2 \pi \sigma}{B}\left(x x^{\prime}+y y^{\prime}\right)\right] \mathrm{d} x^{\prime} \mathrm{d} y^{\prime}
\end{aligned}
$$

When the A coefficient vanishes, we are at a plane where the output field is the Fourier transform of the input field, and when the ' $\mathrm{B}$ ' coefficient vanishes, we are at a plane 
where the output field is the magnified image of the input field. With the constraints in equation (12) still holding, we find that the coefficient ' $A$ ' calculated by matrix multiplication in equation (14) vanished, so we are at a Fourier plane for the aperture stop transmittance at $\mathrm{I}_{0}$ '. Hence, from equation 15 , we find that the PSF is a scaled version of the Fourier transform of $\mathrm{t}(\mathrm{x}, \mathrm{y})$, the aperture stop transmittance, i.e.

$$
h(x, y, \sigma)=T\left(\frac{x}{\beta(\sigma)}, \frac{y}{\beta(\sigma)}\right)
$$

where,

$$
\beta(\sigma)=\frac{B(\sigma)}{\sigma}=M_{0}\left[\frac{D_{0}^{\prime} z \sigma_{0}}{Z_{0} \sigma^{2}}-\frac{D_{0}^{\prime}+z}{\sigma}\right]
$$

Equation (17) gives the scale factor of the PSF as a function wavelength. We can find a position of the aperture plane where the derivative of the scale factor with respect to wave number is zero. At this location, the Fourier transform of the transmittance has the same scale for all wavelengths, and as a result of equation (16) the PSF has the same scale for all wavelengths, assuming all elements are of perfect design. Solving for this aperture plane distance, we find,

$$
D_{0}^{\prime}=\frac{z Z_{0}}{2 z-Z_{0}}
$$

at this location, the scale factor to first order approximation is given as,

$$
\gamma M_{0}=-M_{0}\left(\frac{z^{2}}{\sigma_{0}\left(2 z-Z_{0}\right)}\right)
$$

This is then extended to $2 \mathrm{D}$ objects with electric field distributions over the intermediate image plane which serves as the input plane for the optical correlator. With this, we arrive at our desired result,

$$
U_{\text {out }}(x, y)=U_{\text {in }}\left(\frac{x}{M_{0}}, \frac{y}{M_{0}}\right) * T\left(\frac{x}{M_{0} \gamma}, \frac{y}{M_{0} \gamma}\right)
$$

Equation (20) tells us that our image plane field will be our object plane field convolved with the achromatically scaled Fourier transform of the aperture transmittance function. The bandwidth of the illumination plays no role to first order. If we use polarizationbased phase differences as our aperture transmittance function as those seen in Savart plates and Sagnac shearing interferometers, we will obtain the same result seen in equation 10, but this time, the output is independent of wavelength. 
Temporal coherence effects are simply a manifestation of the chromatically dependent scale of the PSF. These effects are minimized, if the scale of the PSF is achromatized as was done here. In other words, the extended spectrum of the source can be regarded as consisting of many monochromatic components. Each such component generates a perfectly periodic contribution to an arbitrary interferogram, but with a period depending on its particular optical wavelength ${ }^{9}$. By achromatizing the scale factor, we are essentially forcing all wavelengths to have the same period, and thus the visibility reduction inherent with broadband illumination is eliminated to first order with this system.

There is second-order, residual chromatic error in the scale of the PSF, for our system it is given by,

$$
\operatorname{SCE}(\sigma)=100 \frac{\left(\sigma-\sigma_{0}\right)^{2}}{\sigma^{2}}
$$

A plot of this error is given in the following figure:

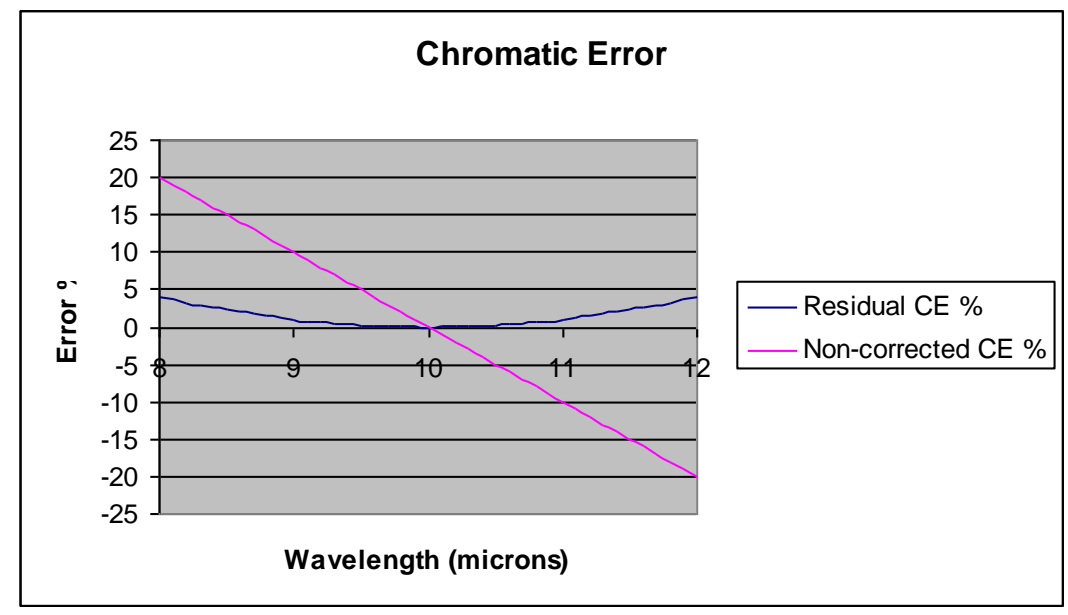

Figure 11 - Second Order Chromatic Error as a function of wavelength.

Figure 11 shows that the PSF is not completely achromatized due to second order effects not specifically treated in the model, but the error induced even under broadband illumination is a factor of 10 or better from the non-corrected system. This sets a limit on how broadband we can make our system and still maintain good fringe visibility. Even so, this instrument allows us to easily use sources of an order of magnitude greater bandwidth than was previously useable. The specific bandwidth to be used would vary on the application.

Now that the coherence properties of the system and the achromatization of the PSF have been theoretically demonstrated, we must design a quality imaging system that can accommodate all of the constraints we've set forth in our models and design.

This system is meant to work in a remote sensing capacity, so we designed the system to work at a distance of 5 miles with the ability to resolve objects 2 feet in diameter. With 
these requirements in mind, we designed a chromatically corrected, $f / 2$ system with an entrance pupil diameter of $100 \mathrm{~mm}$. Again, the system 3D model can be seen in figure 2 .

The front end of the imaging system is a Ritchey-Chretien telescope which provides an intermediate image that acts as the system object for the rest of the design. In keeping with the constraint calculated in equation (18), the object distance equals the first diffractive lens' focal length to approximately collimate the rays as they traverse the two following Sagnac shearing interferometers (SSI's). The focal length and object distance place the aperture approximately in the middle of the two SSI's. What is great about this design is that these parameters can be changed through scale tuning discussed in reference 6 . The distance of the object to the first diffractive lens can be tuned to change the aperture location anywhere we desire to optimize our performance at the image plane.

Again, keeping in accord with the constraints set forth in equation (12), the diffractive elements are simply conjugate elements, the first with positive power and the other with the equal but opposite negative power. This is necessary because the negative dispersion induced past the aperture plane by the first diffractive lens must be canceled out in the image plane to maintain an achromatic PSF. This is accomplished by using the first refractive lens with the conjugate pairs of diffractive lenses. Essentially, the first refractive lens images the first diffractive lens directly onto the second, and since each of them have equal but opposite phase profiles, their overall negative dispersion effect on image quality is canceled. After the second diffractive lens, a second achromatic refractive lens is placed to make the image real and present it to a focal plane array.

Each achromatic refractive lens consists of a Germanium element, with a diffractive optical element placed on the back for efficient color correct, air spaced with a $\mathrm{ZnSe}$ element with an aspheric back surface for complete aberration control. This type of element is essentially completely color corrected with diffraction limited optical performance. Also, this type of element performs well under thermally variant conditions that may be found in mission types where this type of system would be employed.

The system as a whole performs near diffraction limited over the full field of view of 2 degrees. The field of view covers about 281 meters from $8 \mathrm{~km}$. This system can resolve objects 2 feet in diameter from this distance within its field of view. The design suffers from a bit of distortion, but fortunately this type of aberration can be corrected in image processing. The modulation transfer function (MTF) curves for the system follow in figure 12 . 


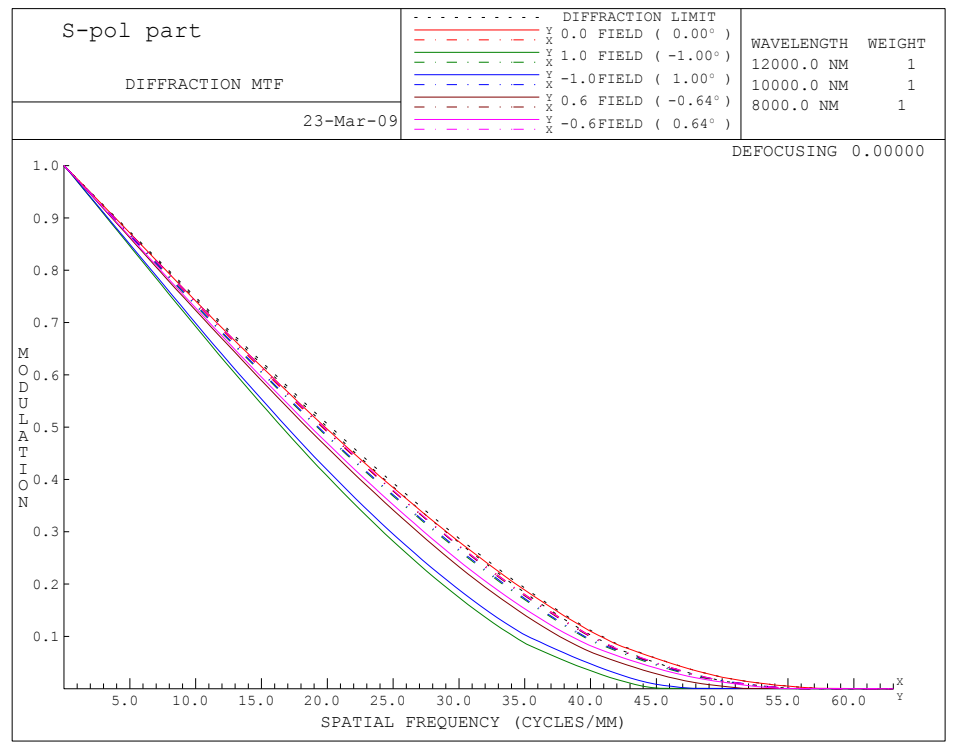

Figure 12 - Modulation Transfer Function curves for the designed system.

The performance illustrated in figure 12 shows that the system performs smooth over all spatial frequencies up to the cut-off frequency. The curves at the full field value don't perform at the diffraction limited level, but they do perform very well and are in keeping with our requirement to be able to resolve objects of 2 feet.

To further demonstrate the system's performance, we performed a $2 \mathrm{D}$ image simulation with the object being an array of USAF 1951 standard resolution targets across the field of view.

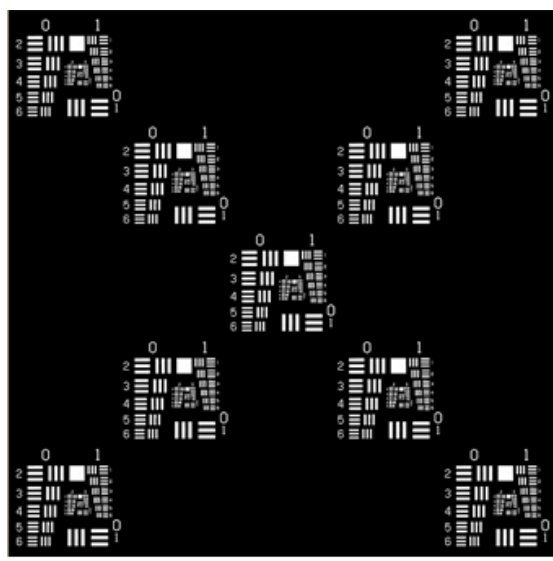

(a)

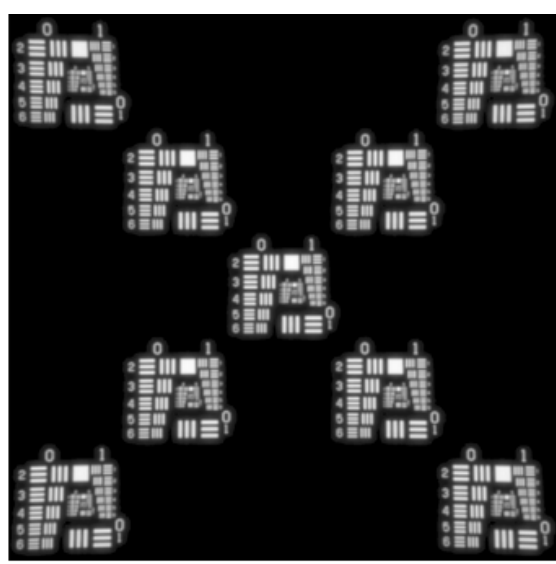

(b)

Figure 13 - 2D image simulation in the LWIR of USAF 1951 resolution target across the field of view. (a) object defined over the full field of view; (b) simulated image.

As was stated earlier, there is distortion evident at the edge of the field and a bit of blurring induced by the residual chromatic error, but when we zoom in to the targets at the edge of the field, we can see how the system performs. Remember, that this target is being simulated over the $281 \mathrm{~m}$ field. 


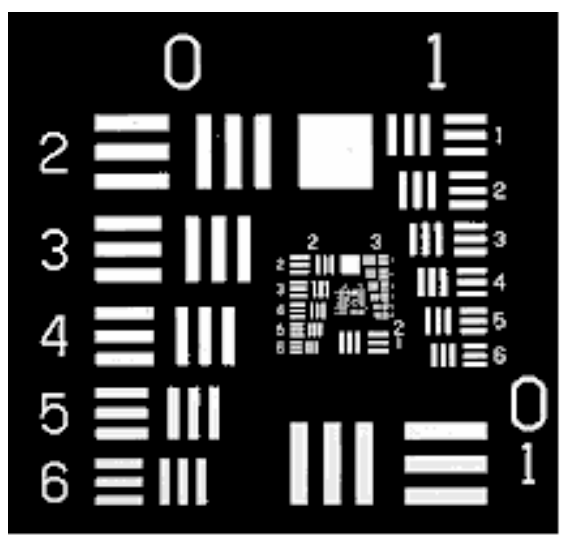

(a)

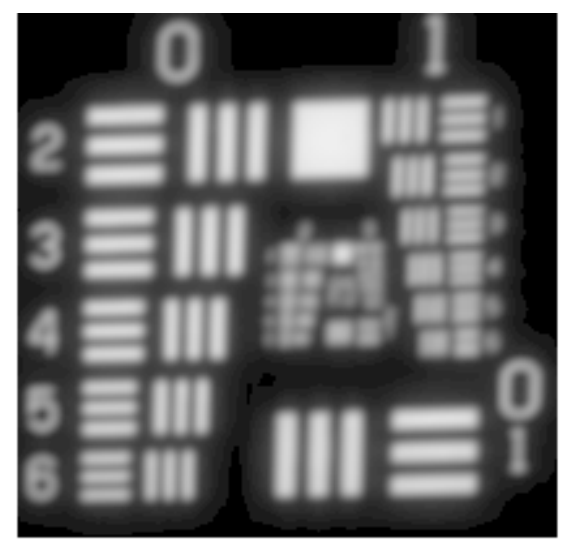

(b)

Figure 14 - Zooming into the edge of the field performance in figure 13. (a) edge of the field object target; (b) edge of the field image simulation.

From this simulation, we can see that the edge of resolution for this system is group 1 element 3. With this system's magnification this corresponds to approximately 0.5 meters/line pair, which is less than 2 feet/line pair.

When we toleranced this system, we found that tight tolerances must be placed on the aspheric A coefficient on the primary and secondary mirror. Other tolerances were set from a default tolerance set by CodeV.

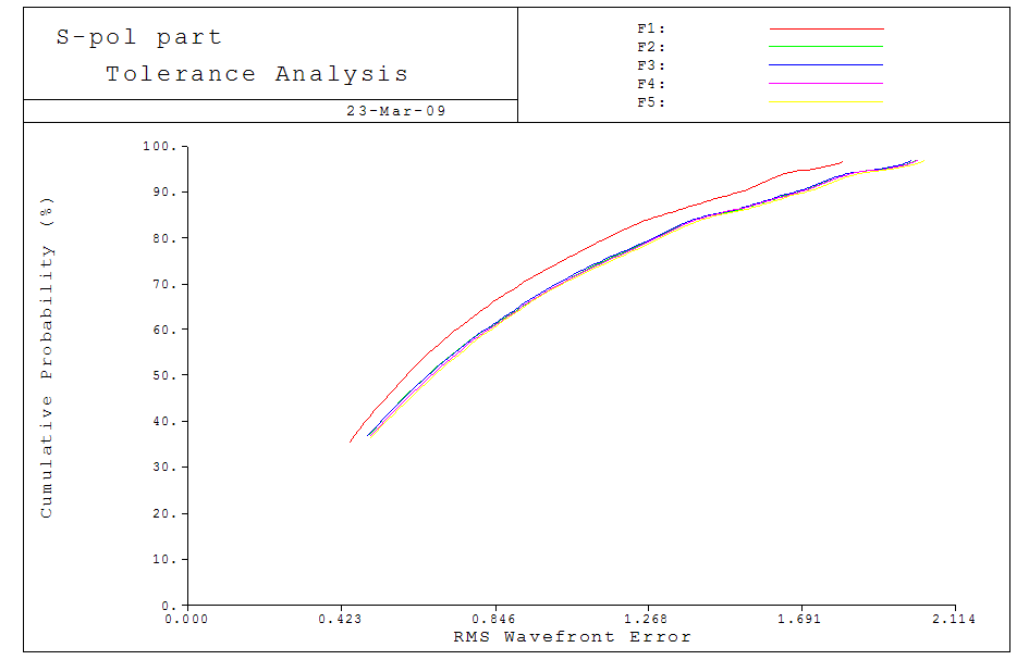

Figure 15 - Tolerance analysis of our designed system. It shows that $97.7 \%$ of the systems will perform with only 1.8 waves of RMS error at the center design wavelength.

With these tolerances, only 1.8 waves of RMS error will be induced for $97.7 \%$ of the designed systems, with less than a wave for the $50 \%$ of the designed systems. The residual RMS wave error was very low, because the system was operating at close do the diffraction limit, but adding 1.8 waves of error would not significantly hinder system performance, and tighter tolerances could be made in other parts of the design to reduce this value, but at a higher cost. 
The system we have presented here will be able to simultaneously achromatically image a remote scene with a full field of view of 2 degrees, and produce an achromatic Fourier transform of its aperture function. The Fourier transform of its aperture function will be convolved with the intermediate image of the scene to spatially modulate objects the end image in such a way that all of the polarization parameters (Stokes vector components) can be gleaned through inverse Fourier transform spectral filtering techniques. It will be able to resolve objects greater than 2 feet in diameter within its field of view.

It should be noted that the above treatments are able to be proven regardless of the spatial coherence of the light over the object plane. As long as we solve the temporal coherence issues associated with the system via AFT, the coherence at the image plane is sufficient to produce high contrast fringes used to obtain the polarization information of objects in the scene.

As was stated earlier, temporal coherence effects are simply a manifestation of the chromatically dependent scale of the PSF. These effects are minimized, if the scale of the PSF is achromatized as was done here. In other words, the extended spectrum of the source can be regarded as consisting of many monochromatic components. Each such component generates a perfectly periodic contribution to an arbitrary interferogram, but with a period depending on its particular optical wavelength ${ }^{9}$. By achromatizing the scale factor, we are essentially forcing all wavelengths to have the same period, and thus the visibility reduction inherent with broadband illumination is eliminated, to first order, with this system.

\section{Section 5. Future Objectives}

The objective of future efforts are to construct, calibrate, and test a prototype of the broadband, thermal infrared snapshot complete imaging Stokes polarimeter as part of a remote sensing system modeled in Phase I. This system will be capable of determining all four Stokes vectors in a snapshot (one integration time) over the LWIR spectral band. First, we need to develop a complete system model based on the theoretical models constructed and optical design completed in Phase I. This complete system model will take into account parameters that weren't treated in the theoretical models of Phase I, such as temperature and transmission of each element. Next, we use the data from the complete system model to assist in the purchase of the system components. Thirdly, we need to characterize, construct, and calibrate each component of the system and the system as whole. Lastly, with the prototype constructed and calibrated, we will take data in the laboratory, and in uncontrolled environments of test subjects with a wide variety of spectral, spatial and polarization profiles. These results will experimentally verify the results found in Phase I and demonstrate the commercial potential for this instrument.

\section{Objective: Model the complete system.}

By combining the theoretical models in Phase I with the optical design of the system, based on the constraints of the system, complete a system model which will include parameters that were not included in the treatments in Phase I. From this model, we can 
compute fringe characteristics of test subjects within the model, such as expected carrier frequencies and overall flux on the detector. With the inclusion of these parameters, we will be able to compute a system level signal to noise ratio (SNR) which will assist in determining the system components. A variety of factors will be addressed, including,

i) How the temperature of each element and the background upon which an object will lie will affect the output.

ii) How the AR coatings of each element will affect the output.

iii) How much cold shielding and individual element cooling will be necessary to obtain good results.

iv) How the inclusion of a generic $\mathrm{HgCdTe}$ detector with specified pixel sizes will affect our results.

\section{Objective: Use the model data to purchase system components.}

During phase I the tolerances and specifications on each component in our system were determined. This information, coupled with the data from the complete system model will allow us to find vendors that can meet our system requirements for each component and purchase the components needed to construct the prototype of the broadband, snapshot, complete imaging Stokes polarimeter.

\section{Objective: Characterize, construct, and calibrate system components.}

The system designed in Phase I consists of many optical elements including aspheric mirrors, flat mirrors, lenses, polarizers and retarders. Characterization of each purchased element's transmission, reflection and polarization characteristics as a function of wavelength and temperature is essential to find any weak links in the optical chain. After characterization is complete of every element in the system, a prototype system will be constructed and aligned on an optical table. Since we are performing interferometric processes, alignment of each element is absolutely critical to maintain good system performance. Finally, we will need to calibrate the entire system with known polarization sources.

\section{Objective: Take data on a variety of sources in a variety of environments.}

With the prototype constructed and calibrated, we will take data in the lab, and in uncontrolled environments of test subjects with a wide variety of spectral, spatial and polarization profiles. These results will experimentally verify the results found in Phase I and demonstrate the capability of this instrument for polarization and spatial measurements. 


\section{Section 6. Specific Future Work Plan}

The broadband LWIR complete imaging Stokes polarimeter based on Sagnac shearing interferometers, shown again below,

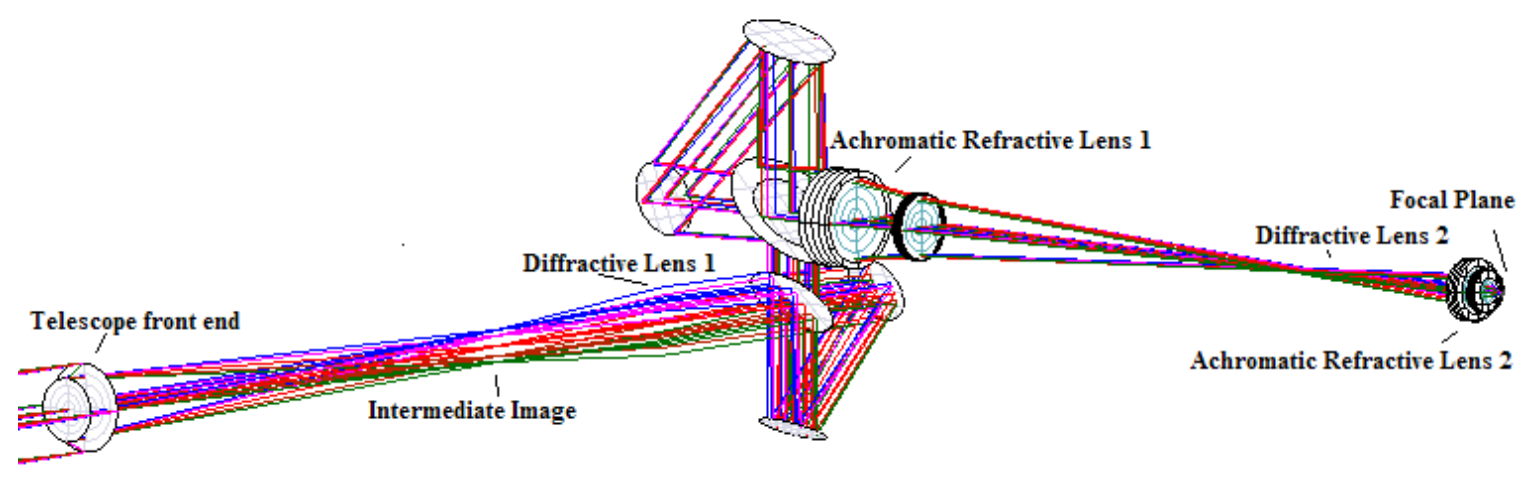

was designed and modeled in phase I of this project. It was determined, through theoretical models that the design would, in fact, produce a spatially modulated image of objects within its field of view. These modulations can be studied; Fourier transformed, and filtered in order to produce the complete polarization content of objects within the scene. This process was first introduced by $\mathrm{Oka}^{3}$. The general irradiance distribution for these spatially modulated scenes are given by ${ }^{3}$,

$$
\begin{aligned}
I(x, y)= & \frac{1}{2} S_{0}(x, y)+\frac{1}{2} S_{2}(x, y) \cos \left[2 \pi U_{2} y\right]-\frac{1}{4}\left|S_{13}(x, y)\right| \cos \left\{2 \pi\left(U_{2}-U_{1}\right) y-\arg \left[S_{13}(x, y)\right]\right\} \\
& +\frac{1}{4}\left|S_{13}(x, y)\right| \cos \left\{2 \pi\left(U_{2}+U_{1}\right) y+\arg \left[S_{13}(x, y)\right]\right\},
\end{aligned}
$$

where, $\quad S_{13}(\sigma)=S_{1}(\sigma)+i S_{3}(\sigma)$,

This is a result matched in our model by equation 10. In general, broadband use of such snapshot imaging polarimeters will produce vastly reduced visibility of the fringes produced by these spatial frequencies, because of the superposition of fringe patterns with different carrier frequencies. In our system, we eliminate this superposition by achromatizing the pupil plane, such that the Fourier transform has the same scale for all spectral components.

Fourier transformation of $\mathrm{I}(\mathrm{x}, \mathrm{y})$ yields seven different carrier frequencies, shown in figure 16, 


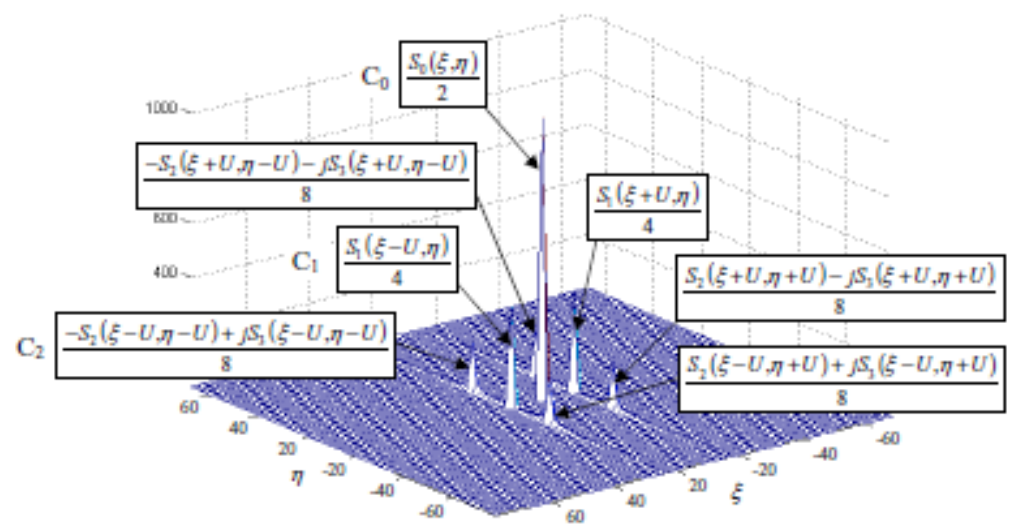

Figure 16 - The Fourier spectra of a general 2D interference pattern produced by a snapshot polarimeter based on channeled polarimetry. Seven channels carrying various portions of the Stokes vector information are present. ${ }^{12}$

The undesired channels are filtered out in Fourier space, and then an inverse Fourier transform is completed to gain the complete Stokes vector of an object within a scene.

The theoretical models given in phase I were based on this concept, but we did not include a few parameters than could affect system performance, such as

v) How the temperature of each element and the background upon which an object will lie will affect the output.

vi) How the AR coatings of each element will affect the output.

vii) How much cold shielding and individual element cooling will be necessary to obtain good results.

viii) How the inclusion of a generic $\mathrm{HgCdTe}$ detector with specified pixel sizes will affect our results.

With these parameters in mind, we need to determine the functional form of the irradiance on the detector due to the temperature of the background and of each element, what AR coating will introduce in the system model as far as Fresnel losses are concerned, what cold shielding and individual element cooling will bring to the model as variables on background and element temperatures, and introduce a generic $\mathrm{HgCdTe}$ detector into the model for sampling measurements.

The irradiance contribution of backgrounds and individual elements in the LWIR are based solely on the object's temperature and emissivity. This irradiance then must pass through successive optical elements with a transmission coefficient. As an example, we model a simple system with a source being imaged through three lens elements with transmission, temperature, and emissivity characteristics, $\tau$, $\mathrm{T}$, and $\varepsilon$ respectively, in the following figure, 


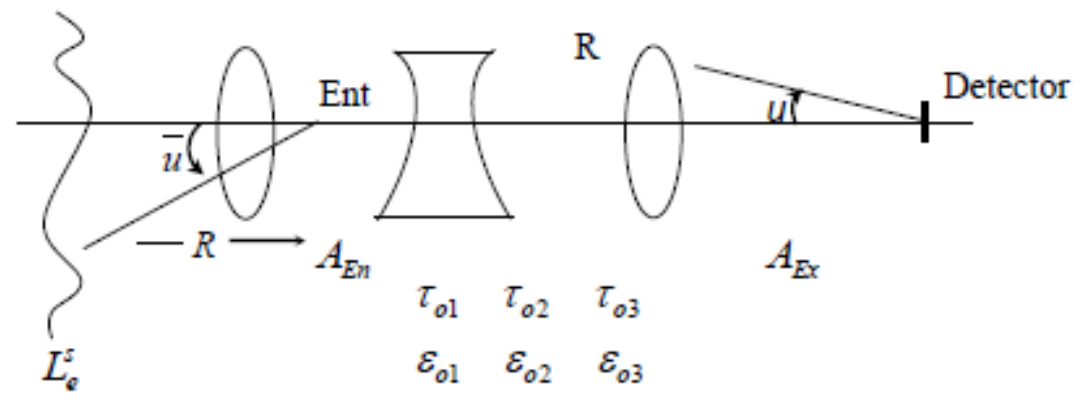

Figure 17 - Sample system to illustrate background irradiance on a detector in the LWIR from lenses ${ }^{13}$.

The irradiance on the detector is a function of the radiance emitted by each element, and the solid angle subtended by that element as seen from the detector, and the transmission of the elements following the element in question. This gives ${ }^{13}$,

$$
\begin{aligned}
E_{e}^{d, o}= & \varepsilon_{o 3} L_{e}^{(3)} \pi \sin ^{2} u+\varepsilon_{o 2} L_{e}^{(2)} \tau_{o 3} \pi \sin ^{2} u \\
& +\varepsilon_{o 1} L_{e}^{(1)} \tau_{o 2} \tau_{o 3} \pi \sin ^{2} u
\end{aligned}
$$

Our system has many more transmissions and reflections than the three shown here, and each polarization of light does not see the same number of each. Therefore, this must be treated in the new, complete system model. Equation 22 shows that the more transmissive our optics are, the greater the background noise will be from the optics, however, we need transmission as high as possible to gain high signal. This treatment also applies to background irradiance at the object plane as well, since each of these elements completely fills the field of view of the detector, as would a background at the source plane.

To ameliorate this issue, we can add a cooling parameter to each lens element and a cold shield at the entrance pupil of the system to cut down on background irradiance at the detector. These are expensive alternatives, but are generally necessary when operating in the LWIR to cut down on background irradiance and increase our SNR. These parameters will easily be added to the model by simply reducing the temperature of the lens elements and the area surrounding the field of view of the detector in the object plane. The amount of cooling needed to attain a good enough SNR to maintain high spatial resolution will be determined within this model and will determine if we must cold shield or perform individual element cooling.

Anti-reflection coatings will also be included in the model at each interface where we will see Fresnel reflections. At normal incidence, the Fresnel reflection coefficient at an air-material interface is,

$$
R=\left(\frac{n-1}{n+1}\right)^{2}
$$


where $\mathrm{n}$ is the index of refraction of the material at a specific wavelength. The index of refraction can be a complex valued quantity, and leads to absorption within the material. As we stray away from normal incidence, the reflection coefficient changes. In any case, an AR coating drastically reduces this reflection coefficient and increases the performance of the interface.

At appropriate locations within the system, we will place AR coating models to study the effect of the AR coatings on different polarizations of incident radiation on the residual reflection coefficients. These effects might include birefringence or transmission differential between orthogonal polarization states. If these effects are present, they will affect the output of the system and must be included in the model and calibrated out in practice as will be discussed later in this section.

In the complete system model, we must also include the effect of atmospheric transmittance. This is relatively simple to do in the LWIR, if we assume no scattering within the atmosphere ${ }^{13}$.

$$
C_{T}=\frac{C(\tau)}{C(100 \%)}=\frac{\text { Contrast with Atm }}{\text { Contrast w/o Atm }}
$$

We wish to know how the transmission of the atmosphere will affect the contrast (MTF) at the image plane. With atmospheric transmittance, $\tau$, we can define a parameter called the contrast transmittance, given in equation $(24)^{13}$. Because scattering can be neglected in the IR, the contrast transmittance, $C_{T}=\tau$. This gives a simple way to model the affect of atmospheric transmittance. The basic system block diagram for this situation goes as follows ${ }^{13}$,

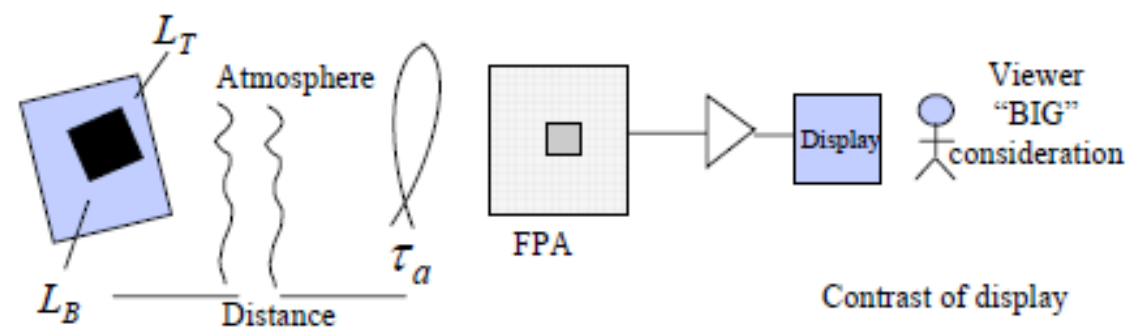

Figure 18 - Block diagram for affect of atmospheric transmission when viewing targets on a background. ${ }^{13}$

Within figure 18, we see the Focal plane array (FPA). This part of the system plays a large role in how the scene is displayed. It introduces different types of noise and samples the image in such a way that can only reduce the overall image quality.

Using a simple $\mathrm{HgCdTe}$ model with pixel sizes indicative of industry standards $(5 \mu \mathrm{m})$, we will include how this type of detector will reduce the image contrast and lower the SNR in our model. 


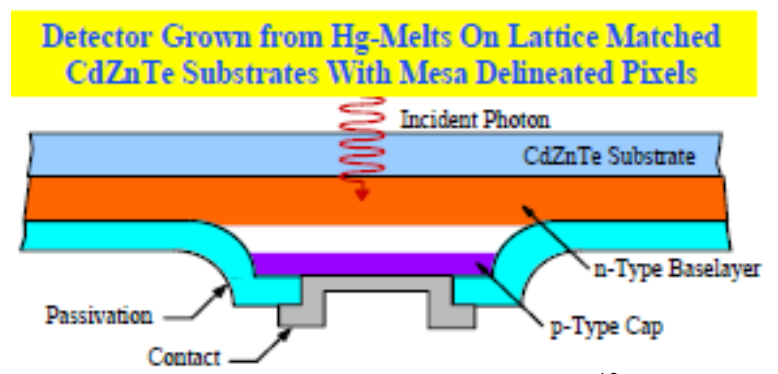

Figure $19-\mathrm{HgCdTe}$ structure. ${ }^{13}$

The primary sources of noise in this detector with a preamplifier are shot noise of the reverse saturation current; photon noise; Johnson noise of the load resistor and detector resistance; $1 / \mathrm{f}$ noise, and preamplifier noise ${ }^{14}$. These noises are summed in square as ${ }^{14}$,

$$
i_{n}^{2}=4 q^{2} i_{0} \Delta f+2 q^{2} \eta\left(\phi_{q, s i g}+\phi_{q, b k g}\right) \Delta f+\frac{4 k T_{d} \Delta f}{R_{d}}+\frac{4 k T_{f} \Delta f}{R_{f}}+\frac{\beta_{0} \bar{i} \Delta f}{f}+\bar{i}_{p a}^{2}+\bar{v}_{p a}^{2}\left(\frac{R_{f}+R_{d}}{R_{f} R_{d}}\right)^{2}
$$

where, $\mathrm{q}$ is the charge of an electron, $\mathrm{i}_{0}$ is the reverse saturation current, $\Delta \mathrm{f}$ is the electrical bandwidth, $\eta$ is the quantum efficiency, $\phi_{q, s i g}$ and $\phi_{q, b k g}$ are the signal and background photon fluxes, $\mathrm{k}$ is Boltzmann's constant, $\mathrm{R}_{\mathrm{d}}$ and $\mathrm{R}_{\mathrm{f}}$ are the detector and feedback resistances, $T_{d}$ and $T_{f}$ are the detector and feedback resistor temperatures, $f$ is the electrical chopping frequency, $i$ is the dc current through the detector, $i_{p a}$ is the preamplifier current noise, and $\mathrm{v}_{\mathrm{pa}}$ is the preamplifier voltage noise.

With equation 25, we find the SNR of the system to be,

$$
\frac{S}{N}=\frac{q \eta \phi_{q, s i g}}{i_{n}}
$$

With the inclusion of all the above parameters for the cold shielding, individual element cooling, detector noise, background irradiance, AR coatings and atmospheric transmission into the models we used in phase I, we can find a much more accurate representation of system performance that can be used to assist in the determination of vendors for technical objective $B$. These determinations will also assist in deciding if cooling individual components and cold shielding are necessary to maintain good system performance.

With the data from the activities described above, we will reestablish our system tolerances and make choices as to what cooling mechanisms will be necessary to maintain good system performance from a variety of modeled test subjects that would be targets indicative of polarized objects with unpolarized backgrounds and any permutations thereof. 
We have found vendors for all of the system components for the tolerances set in Phase I, but they are not all of U.S. origin. Many of the system component tolerances in Phase I will still apply. Those components that may have their tolerances change after activities described above are completed will wait until the conclusion of modeling activities, then vendors for those parts will be sought out. These components include, but are not limited to, cold shields and cooling mounts.

To reiterate, the next objective is to characterize each system component, construct the system to specification outlined in the optical design, and to calibrate the system in order to optimize the performance.

The entire system consists of two aspheric mirrors, two diffractive lenses, six flat mirrors, three half-wave retarders, five linear polarizers, and two air-spaced achromatic doublets with a $\mathrm{HgCdTe}$ focal plane array. Each of these elements must be characterized as far as transmission characteristics, reflective characteristics, and polarization characteristics as a function of wavelength.

Each of these characteristics will be calculated with a LWIR Fourier transform spectrometer (FTS) available in our laboratory. Fourier transform spectroscopy is a measurement technique where the spectrum of a source is collected through measuring the form of the temporal coherence (fringe visibility) of a source being interfered in an interferometer. In our FTS, the interferometer being used is a typical Twyman-Green interferometer to observe the functional form of the visibility of the interferogram as the OPD is changed in the interferometer. The following equations give a short theoretical treatment on out the FTS works, with the power spectrum of the source given as $\mathrm{a}^{2}(\mathrm{v})$, and the visibility given as $\mathrm{V}(\Delta \mathrm{t})$.

$$
\begin{aligned}
& V(\Delta t)=\Im\left[a^{2}(v)\right] \\
& \Delta t=\frac{O P D}{c} \\
& \therefore a^{2}(v)=\mathfrak{J}^{-1}\left[V\left(\frac{O P D}{c}\right)\right]
\end{aligned}
$$

The Fourier transform of the power spectrum is the visibility as a function of the OPD in the interferometer. Therefore, the power spectrum is simply the inverse Fourier transform of the visibility that is observed.

To measure the transmission of each element, the element will be placed following the FTS source, so that all radiation that would normally go into the FTS will first go through the element. Similarly, to measure the reflection of each element, the source will be made to reflect off of the element, then enter the entrance pupil of the FTS. In these configurations, the transmission as a function of wavelength, $t(\lambda)$, and reflection as a function of wavelength, $\rho(\lambda)$, will be measured. As a result of these measurements, we will also obtain the emissivity of each element. 
To obtain each element's spectral polarization characteristics, we will use the FTS as the detector behind a system that will be able to determine the complete Mueller matrix of an optical element. As the front end polarizer and retarder are rotated, the polarization state incident on the test element can be changed. The test element will then act on that polarization state, next the radiation will be sent through an analyzer retarder and polarizer and into the FTS detector as seen in figure 20.

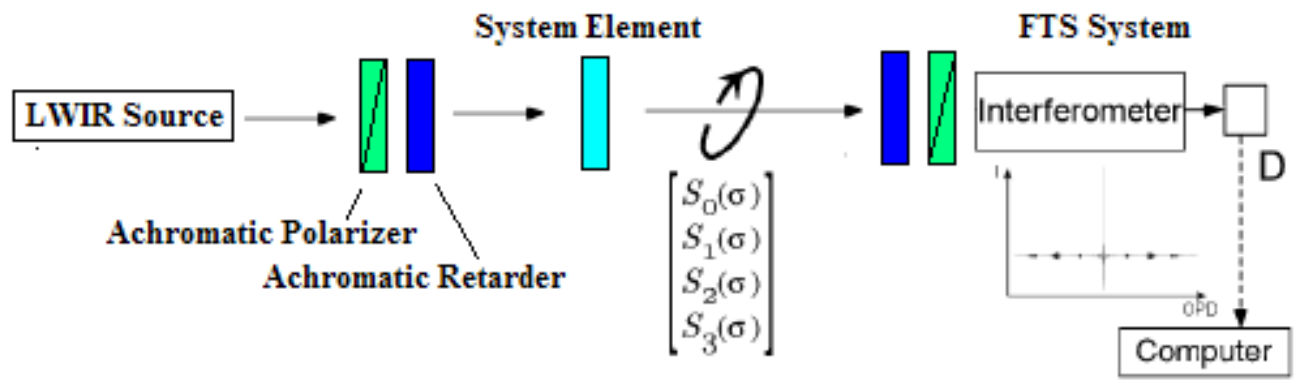

Figure 20 - Element characterization scheme with a FTS as the detector.

In this configuration, every Mueller matrix element of the tested system element can be determined. When each Mueller matrix element for the tested system element is determined, the element's spectral polarization characteristics will be known ${ }^{11}$. The FTS system will measure irradiance, $\mathrm{I}(\lambda)$ in the following equation,

$$
\left(\begin{array}{c}
I(\lambda) \\
\bullet \\
\bullet \\
\bullet
\end{array}\right)=\vec{a} \tilde{M} \vec{p}=\left[\begin{array}{cccc}
a_{1}(\lambda) & a_{2}(\lambda) & a_{3}(\lambda) & a_{4}(\lambda) \\
\bullet & \bullet & \bullet & \bullet \\
\bullet & \bullet & \bullet & \bullet \\
\bullet & \bullet & \bullet & \bullet
\end{array}\right]\left(\begin{array}{llll}
m_{11}(\lambda) & m_{12}(\lambda) & m_{13}(\lambda) & m_{14}(\lambda) \\
m_{21}(\lambda) & m_{22}(\lambda) & m_{23}(\lambda) & m_{24}(\lambda) \\
m_{31}(\lambda) & m_{32}(\lambda) & m_{33}(\lambda) & m_{34}(\lambda) \\
m_{41}(\lambda) & m_{42}(\lambda) & m_{43}(\lambda) & m_{44}(\lambda)
\end{array}\right)\left[\begin{array}{c}
p_{1}(\lambda) \\
p_{2}(\lambda) \\
p_{3}(\lambda) \\
p_{4}(\lambda)
\end{array}\right]
$$

where $\mathbf{a}(\lambda)$ is the Mueller matrix of the analyzer polarizer and retarder combination, $\mathbf{M}(\lambda)$ is the Mueller matrix for the test element we wish to determine, and $\mathbf{p}(\lambda)$ is the product of the Stokes vector of the light from the source with the Mueller matrix of the polarization state generator given by the combination of the first polarizer and retarder ${ }^{11}$.

With a normal detector, only $\mathrm{I}_{0}$ is measured, this is why only the first row of the analyzer Mueller matrix is necessary to know, because they are the only elements involved in determining $\mathrm{I}_{0}$. If a unique set of 16 independent measurements are made, the Mueller matrix of the test element at each wavelength within our spectral band can be determined.

To compute the entire spectral Mueller matrix of the test element, we first write the Mueller matrix in a 16x1 vector instead of the normal $4 \times 4$ form. We then, define a new $1 \mathrm{x} 16$ vector for each measurement condition set by the analyzer and generator polarizers. For the qth measurement, this vector is defined as, 


$$
\begin{aligned}
& \vec{W}_{q}=\left(\begin{array}{lllllll}
w_{q, 11}(\lambda) & w_{q, 12}(\lambda) & w_{q, 13}(\lambda) & w_{q, 14}(\lambda) & w_{q, 21}(\lambda) & \ldots & w_{q, 44}(\lambda)
\end{array}\right) \\
& \vec{W}_{q}=\left(\begin{array}{lllllll}
a_{q, 1} p_{q, 1} & a_{q, 1} p_{q, 2} & a_{q, 1} p_{q, 3} & a_{q, 1} p_{q, 4} & a_{q, 2} p_{q, 1} & \ldots & a_{q, 4} p_{q, 4}
\end{array}\right)
\end{aligned}
$$

We will take 16 separate, unique measurements which will define another matrix $\mathbf{W}$. This new matrix will be a $16 \times 16$ matrix where each row is a separate $\mathrm{W}_{\mathrm{q}}$ vector that corresponds to the irradiance measurement that was generated from the applicable measurement condition. Equation 29 describes the previous statement in matrix form ${ }^{11}$,

$$
\vec{I}(\lambda)=\tilde{W} \vec{M}=\left[\begin{array}{c}
I_{1}(\lambda) \\
I_{2}(\lambda) \\
\vdots \\
I_{16}(\lambda)
\end{array}\right]=\left[\begin{array}{cccc}
w_{1,11}(\lambda) & w_{1,12}(\lambda) & \cdots & w_{1,44}(\lambda) \\
w_{2,11}(\lambda) & w_{1,12}(\lambda) & \cdots & w_{1,44}(\lambda) \\
\vdots & & & \vdots \\
w_{16,11}(\lambda) & w_{16,12}(\lambda) & \cdots & w_{1,44}(\lambda)
\end{array}\right]\left[\begin{array}{c}
m_{11} \\
m_{12} \\
\vdots \\
m_{44}
\end{array}\right]
$$

solving for $\mathbf{M}$,

$$
\vec{M}(\lambda)=\tilde{W}^{-1}(\lambda) \vec{p}(\lambda)
$$

For each spectral component we will receive a Mueller matrix that will characterize the test element's polarization action. For a true broadband component, the Mueller matrix will be independent of wavelength and will be equal for through the entire LWIR spectrum.

After characterization is complete of every element in the system, a prototype system will be constructed and aligned on an optical table. Since we are performing interferometric processes, alignment of each element is absolutely critical to maintain good system performance.

First, each Sagnac interferometer will be aligned with the polarization elements removed, independent of each other element of the designed system given in figure 2. Initially, the first Sagnac interferometer will be aligned by sending collimated, coherent radiation from a small extended source through the system as seen in figure 5a. A lens will be placed after the output mirror to produce fringes. These fringes will then be completely eliminated by changing the' $d$ ' parameter seen in figure 4 . When they are eliminated, the first Sagnac is in a zero OPD state and we move on to the second Sagnac and repeat the process.

Once the Sagnacs are in zero OPD states, each other element will be placed in approximate locations using a gut ray that will be sent through the system with a small collimated beam, such as that from a laser. Each element will then be moved in $\mathrm{x}, \mathrm{y}, \mathrm{z}, \Theta$, and $\varphi$. By studying how the gut ray moves in reflection from the front surface of each element at the source, we can determine if it is in the correct location as far as tilts and movements normal to the optical axis go. Optical axis locations will be determined through intermediate imaging relations. 
Once the system is characterized and constructed, a calibration procedure will be constructed in order to optimize system performance and correct for known errors of each system element.

Polarimeters used in the infrared spectrum must correct for detector offsets and emission from the optics being used, because they are also emitters in the LWIR. The amount of emission is based on each element's temperature. In Michael Kudenov's paper ${ }^{12}$, "Prismatic imaging polarimeter calibration for the infrared spectral region," he treats the calibration procedure for the system given in the following figure,

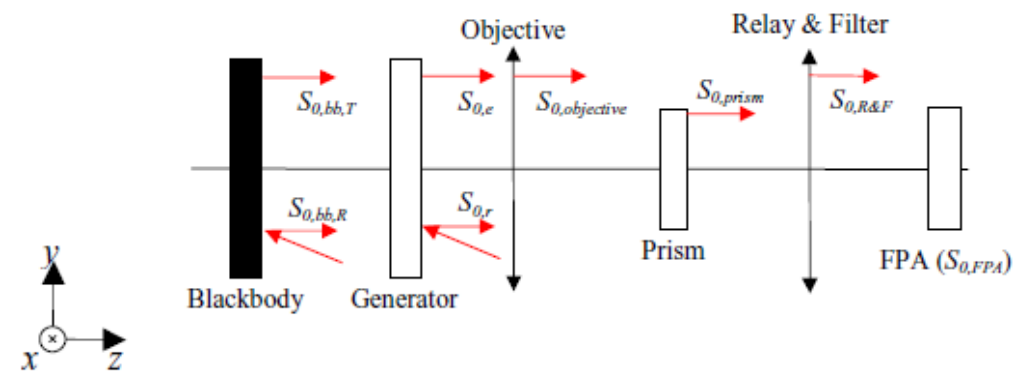

Figure 21 - Prismatic imaging polarimeter calibration setup.

This type of polarimeter is very similar to our system, because it too uses a spatially modulated image to back out the Stokes parameters. The basic system concept as far as the imaging polarimetry is the same, so calibration procedures for each will parallel each other.

To treat the radiometric calibration of the system, he looks at energy emitted and reflected from each element. Kudenov states, "The optics also emit energy (SO,objective, $S O$, prism, and $S O, R \& F$ ) in addition to the FPA's analog to digital conversion offset ( $S O, F P A)$. It should be noted that the reflected term is included for the blackbody since, in practice, these never have an ideal emissivity of 1.0."12

First, a reference measurement is taken of just the blackbody through the generator; this reference data must be removed. It is given as,

$$
\begin{aligned}
& S_{0, \text { total }}=S_{0, b b, T}+S_{0, b b, R}+S_{0, r}+S_{0, e}+S_{0, \text { optics }}+S_{0, \text { objective }} \\
& \text { where, } \quad S_{0, \text { optics }}=S_{0, \text { prism }}+S_{0, R \& F}+S_{0, F P A}
\end{aligned}
$$

He then takes reference measurements at two different blackbody temperatures. Subtracting these two measurements removes offsets induced by a few of the terms. The resulting intensity pattern is proportional to the difference in the blackbody terms. ${ }^{12}$

A condition for calibration is that the normalized Stokes vector from the difference of the two temperatures is equal to the original normalized inputs, states Kudenov. In his 
treatment, he finds that this is true in his system, because of a linear increase in measurements with temperature.

To ensure the offsets are independent of the sample data take after the generator and blackbody are removed, we must use an appropriate experimental procedure ${ }^{12}$. After Kudenov uses a specific experimental procedure, he finds an offset irradiance distribution that is independent from the sample data. By subtracting the offset data from sample data, he finds ${ }^{12}$,

$$
\begin{aligned}
& I_{\text {sample }}-I_{\text {offset }}=\left(S_{0, \text { sample }} \gamma+S_{1, \text { sample }} \varepsilon\right)+\left(S_{0, \text { sample }} \varepsilon+S_{1, \text { sample }} \gamma\right) \cos (2 \pi U x) \\
& \quad+\left|S_{23, \text { sample }}\right|\left[\cos \left(2 \pi U(x+y)+\arg \left[S_{23, \text { sample }}\right]\right)-\cos \left(2 \pi U(x-y)-\arg \left[S_{23, \text { sample }}\right]\right)\right]
\end{aligned}
$$

This is an important result in that, after going through the calibration procedure, he finds that he can find an offset value such that when subtracted from his sample data, he only has terms dependent on the characteristics of the sample. Furthermore, his final answer is exactly in the form of equation 10, the result of our Jones matrix system modeling. It shows that all of the Stokes parameters are modulated by individual carrier frequencies. This allows us to simultaneously image and obtain polarization information in the scene in a snapshot via inverse Fourier transform using Sagnac shearing interferometers in place of Savart plates or prisms. This also agrees heavily with equation 1, a result that was proven experimentally by Luo and $\mathrm{Oka}^{8}$. The parameters, $\varepsilon$ and $\gamma$, are simply scale parameters indicative of the calibration technique.

In his paper, Kudenov, also treats dichroism and Fresnel losses at the prism. In their specific polarimeter, they could not use AR coatings at the prism interfaces, because it hampers system performance by changing the index at the prism interface. Our design will be able to use AR coatings at virtually every interface where they are applicable. We still, however, might see a small degree of dichroism at our beam splitters and other interfaces. We also might expect a small degree of Fresnel losses at lens interfaces.

With a calibrations completed for radiometric offsets, dichroism, and Fresnel losses, he shows raw data sets followed by calibrated data sets for the following system setup,

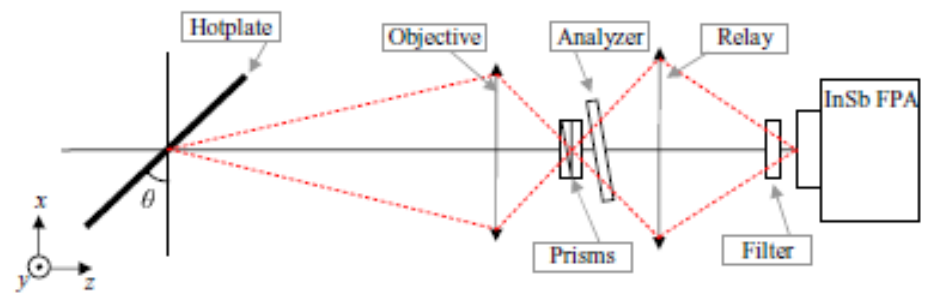

Figure 22 - Birefringent prism imaging polarimeter experimental setup for accuracy assessment. ${ }^{12}$

In his experiment, the hotplate was rotated in 10 degree increments where, at each increment, raw data is taken. From this raw data, the degree of linear polarization (DOLP) is calculated through channeled polarimetry, after calibration. 
Figure 23 demonstrates the feasibility of his calibration technique. The raw data shows that when the hot plate is at 0 degrees, there are fringes, where there shouldn't be, theoretically. These fringes are do to dichroism, Fresnel losses at the prism, and radiometric offsets of the entire system. However, when the DOLP is calculated, the calibration procedure eliminates the DOLP that would be detected without the calibration.
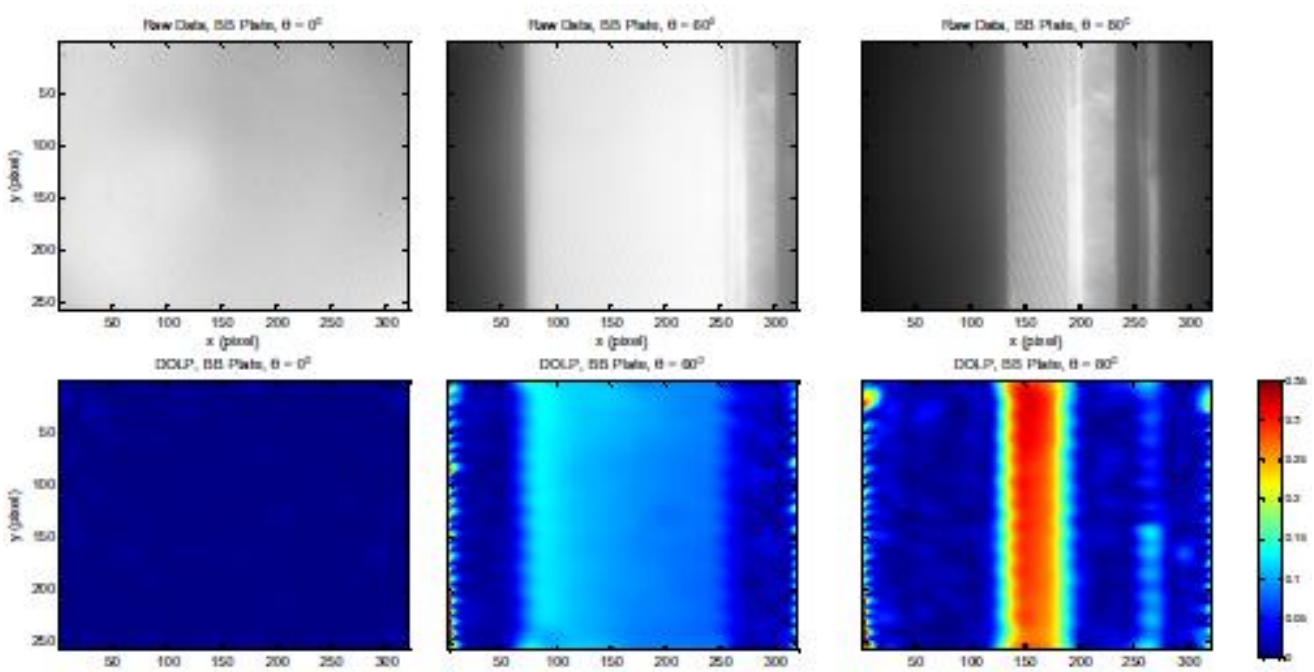

Figure 23 - Raw data in the top row, and DOLP in the bottom row for the tilted plate with the improved calibration $^{12}$.

Our calibration techniques will closely mirror those presented in ref[12], because the systems are very similar and demonstrate many of the same issues, because of the wavelength band in which the system is used. It is expected that Kudenov's calibration techniques will also vastly increase the output of our system.

With the prototype constructed and calibrated, we will take data in the lab, and in uncontrolled environments of test subjects with a wide variety of spectral, spatial and polarization profiles. These results will experimentally verify the results found in Phase I.

The laboratory resources at the University of Arizona includes many different sources of differing spatial, spectral, and polarization based profiles, because of our extensive work in the IR, and these materials are readily available to us. For example, in Kudenov's paper on the calibration of the prismatic imaging polarimeter, he used a spherical blackbody and achieved the following data, 


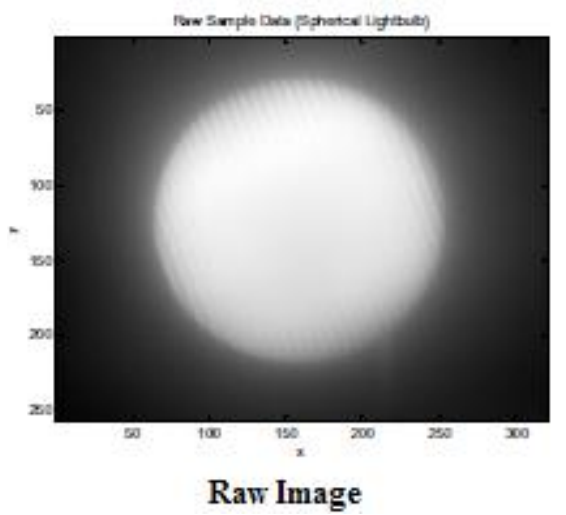

Figure 24- Raw image taken by Kudenov of a spherical black body. ${ }^{12}$

After taking the raw image, he used the data reconstruction algorithms and calibration techniques discussed earlier and throughout this proposal to achieve the following data,

(a)

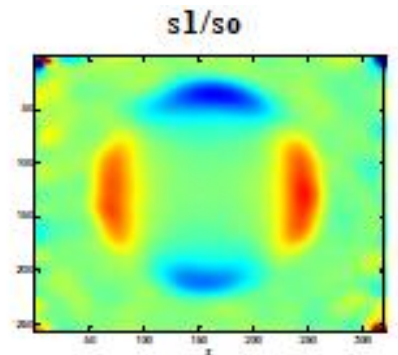

DOLP

(b)

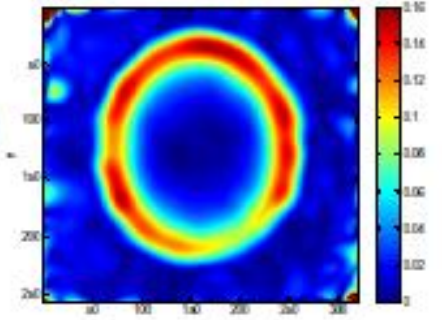

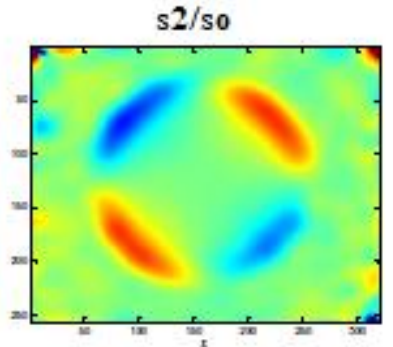

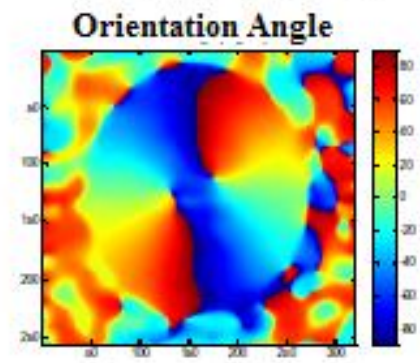

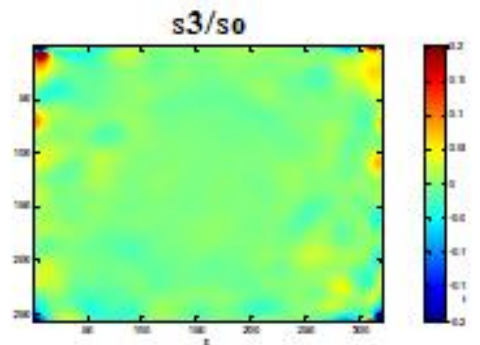

Ellipticity Angle

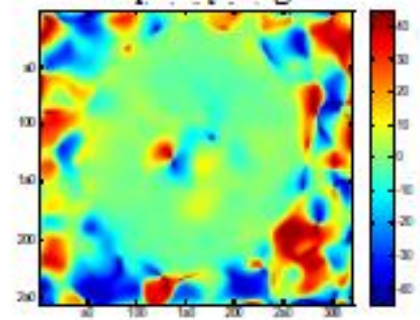

Figure 25 - (a) reconstructed Stokes vector elements normalized by $\mathrm{s}_{0}$. (b) Degree of linear polarization, orientation angle, and ellipticity angle of the polarized radiation emitting from the spherical BB.

These are the type of data products that will be produced by our system when viewing polarized objects. Michael Kudenov is a member of our research group at the University of Arizona and completed his measurements there, as will our team working on this project.

We will take data on many more objects than just a single spherical blackbody. We will also attempt to ruggedize the system in order to make it portable and take measurements on objects in a real-world environment. This will only take place after a large set of data from the lab environment has been completed. Ruggedization of this instrument will consist of manually reconstructing the instrument on a portable mount that can hold all of the system's instrumentation.

It is anticipated that this system will perform well in all environments and produce accurate data products similar to those shown in figure 25 over a 2 degree field of view, 
meant for remote sensing purposes. This system will be able to take these measurements over large spectral bands in the LWIR region, because of the achromatization of the Fourier transform of the pupil function demonstrated in Phase I. This sensor data should experimentally verify the theoretical models completed in Phase I, and lend itself to extension to commercial development.

The independent technologies discussed here in phase I are well documented. By combining the concept of the Savart Plate imaging snapshot polarimeter, the white light correlator, and calibration and characterization techniques, we will develop and produce a broadband, snapshot LWIR Sagnac interferometer imaging polarimeter for use in remote sensing applications that will produce accurate data products of objects within its field of view.

\section{Section 7. Equipment}

In order to construct a prototype, we must purchase the following items listed in table 2,

\begin{tabular}{|c|c|c|c|}
\hline Component & \# of Units & Cost per unit & Total Cost \\
\hline LWIR Polarizers & 5 & $\$ 1,600.00$ & $\$ 8,000.00$ \\
\hline LWIR Beam Splitter & 2 & $\$ 2,000.00$ & $\$ 4,000.00$ \\
\hline LWIR Half-Wave Plates & 3 & $\$ 5,000.00$ & $\$ 15,000.00$ \\
\hline LWIR Refractive Achromats & 2 & $\$ 7,500.00$ & $\$ 15,000.00$ \\
\hline Diffractive Lenses & 2 & $\$ 2,500.00$ & $\$ 5,000.00$ \\
\hline LWIR Telescopic Front End & 1 & $\$ 15,000.00$ & $\$ 15,000.00$ \\
\hline Flat Mirrors & 4 & $\$ 150.00$ & $\$ 600.00$ \\
\hline Mounting Accessories & 1 & $\$ 5,000.00$ & $\$ 5,000.00$ \\
\hline \multirow[t]{2}{*}{ Focal Plane } & 1 & $\$ 25,000.00$ & $\$ 25,000.00$ \\
\hline & & Total Costs: & $\$ 92,600.00$ \\
\hline
\end{tabular}

Each of the items listed in table 2 are the minimum amount of units necessary to fully construct the designed prototype. Not all of the vendors for which we obtained these quotes were American in origin. These costs may fluctuate based on American availability of these elements. 


\section{References}

1. Tyo, S., D. Goldstein, D. Chenault, and J. Shaw, "Review of passive imaging polarimetry for remote sensing applications," Applied Optics, Vol. 45, No. 22(2006) pp.5453-5469.

2. Walraven R. "Polarization imagery," Optical Engineering, Vol. 20, No. 1 (1981) pp. 014-018.

3. Oka, K., and N. Saito, "Snapshot complete imaging polarimeter using Savart Plates," Proc. Of SPIE, Vol. 6295, Infrared Detectors and Focal Plane Arrays VIII (2006).

4. Jones, D. G., D. H. Goldstein, and J. C. Spaulding, "Reflective and polarimetric characteristics of urban materials," Proc. SPIE, Col 6240, Polarization:

Measurement, Analysis, and Remote Sensing VII (2006)

5. Chung, J., et al. "in vivo imaging polarimeter for oral precancer detection," Proc. Of SPIE, Vol. 5693, Optical Tomography and Spectroscopy of Tissue VI (2005).

6. place

7. J. Ferrari and E. Garbusi, "Phase-shifting (Sagnac) interferometer with external phase control," Appl. Opt. 44, 4510-4512 (2005)

8. H. Luo, K. Oka, E. DeHoog, M. Kudenov, J. Schiewgerling, and E. Dereniak, "Compact and miniature snapshot imaging polarimeter," Appl. Opt. 47, 44134416 (2008).

9. J. W. Goodman, Statistical Optics, (John Wiley and Sons, Inc., 1985) chap. 5, pp. 159-161.

10. J. A. Shaw, "Degree of linear polarization in spectral radiances from waterviewing infrared polarimeters," Appl. Opt. 38, 3157-3165 (1999).

11. D. Goldstein, Polarized Light, $2^{\text {nd }}$ Edition, (Marcel Dekker, 2003), chap. 28, pp. 559-576.

12. M. W. Kudenov, L. Pezzaniti, E. Dereniak, and G. Gerhart, "Prismatic imaging polarimeter calibration for the infrared spectral region," Opt. Express 16, 1372013737 (2008)

13. E. Dereniak, D. Staplebrook, Opti 566 Class Notes, Spring '09, University of Arizona.

14. E. Derniak, G. Boreman, Infrared Detectors and Systems, (John Wiley and Sons, Inc., 1996), chap. 7, pp. 264-266. 